\title{
Mooring System Diagnosis and Structural Reliability Control for Position Moored Vessels
}

\author{
Fang, Shaoji; Blanke, Mogens; Leira, Bernt J.
}

Published in:

Control Engineering Practice

Link to article, DOI:

10.1016/j.conengprac.2014.11.009

Publication date:

2015

Document Version

Early version, also known as pre-print

Link back to DTU Orbit

Citation (APA):

Fang, S., Blanke, M., \& Leira, B. J. (2015). Mooring System Diagnosis and Structural Reliability Control for Position Moored Vessels. Control Engineering Practice, 36, $12-26$.

https://doi.org/10.1016/j.conengprac.2014.11.009

\section{General rights}

Copyright and moral rights for the publications made accessible in the public portal are retained by the authors and/or other copyright owners and it is a condition of accessing publications that users recognise and abide by the legal requirements associated with these rights.

- Users may download and print one copy of any publication from the public portal for the purpose of private study or research.

- You may not further distribute the material or use it for any profit-making activity or commercial gain

- You may freely distribute the URL identifying the publication in the public portal 


\title{
Mooring System Diagnosis and Structural Reliability Control for Position Moored Vessels
}

\author{
Shaoji Fang ${ }^{\mathrm{a}}$, Mogens Blanke ${ }^{\mathrm{d}, \mathrm{b}}$, Bernt J. Leira ${ }^{\mathrm{c}}$ \\ ${ }^{a}$ Subsea Installations, Technip Norge A/S, NO 1303 Sandvika, Norway \\ ${ }^{b}$ Department of Electrical Engineering, Technical University of Denmark, DK 2800 Kgs. Lyngby, Denmark \\ ${ }^{c}$ Department of Marine Technology, Norwegian University of Science and Technology, NO 7491, Norway \\ ${ }^{d}$ AMOS CoE, Institute for Technical Cybernetics, Norwegian University of Science and Technology, NO 7491, Norway
}

\begin{abstract}
Early diagnosis and fault-tolerant control are essential for safe operation of floating platforms where mooring systems maintain vessel position and must withstand environmental loads. This paper considers two critical faults, line breakage and loss of a buoyancy element and employs vector statistical change detection for timely diagnosis of faults. Diagnosis design is scrutinized and a procedure is proposed based on specified false alarm probability and estimation of the distribution of the test statistics on which change detection is based. A structural reliability index is applied for monitoring the safety level of each mooring line and, a set-point chasing algorithm accommodates the effects of line failure, as an integral part of the reliabilitybased set-point chasing control algorithm. The feasibility of the diagnosis and of the fault-tolerant control strategy is verified in model basin tests.
\end{abstract}

Keywords: Fault diagnosis, Fault-Tolerant Control, Position Mooring, Structural Reliability, Change Detection, Optimal Set-point Chasing

$\begin{array}{ll}\text { Nomenclature } \\ \text { DP } & \begin{array}{l}\text { Dynamic Positioning } \\ \text { FPSO }\end{array} \\ & \begin{array}{l}\text { Floating production } \\ \text { storage and offloading }\end{array} \\ \text { LF } & \text { Low frequency } \\ \text { WF } & \text { Wave frequency } \\ \text { MSS } & \text { Marine system simulator } \\ \text { IMO } & \text { International Maritime Organisation } \\ \text { PM } & \begin{array}{l}\text { Position mooring with } \\ \text { thruster assistance }\end{array} \\ \text { COT } & \text { Centre of turret } \\ \text { COG } & \text { Centre of gravity } \\ \text { HRS } & \text { Hydro-acoustic reference system } \\ \text { MRU } & \text { Motion reference units } \\ \text { EFF } & \text { Earth-fixed frame } \\ \text { BFF } & \text { Body-fixed frame } \\ \text { TP } & \text { Terminal point } \\ \text { MLE } & \text { Maximum likelihood estimation }\end{array}$

\section{Introduction}

Increasing safety and efficiency of the work-over operation on floating platforms is attracting more and more attention. In offshore operations, marine vessels are often required to be kept at a position by thrusters, known as a DP system. References to DP systems can be found in [1], [2] and [3] etc. For dynamic positioning of surface vessels moored to the seabed, this is referred to as a PM system. The main objective of the PM system is to keep the vessel at a certain position, while the secondary objective is to prevent line breakage by keeping the vessel within a limited region. Several studies have appeared about thruster-assisted PM systems, including [4], [5], [6] and [7], and reliability issues were addressed together with the DP control aspects in [8]. The reactions in the event of faults and the ability to maintain safe control even in case of failure of part of a mooring system has only been sparsely dealt with in earlier studies and this will be the main subject of this paper.

Fault-tolerant control of marine vessels presents 
a challenging issue. Regulations normally define different system levels by hardware redundancies and prevent system failure by replacing faulty hardware [9]. Risk analysis is also performed to evaluate the effect of faults, which is traditionally based on the reliability characteristics of mechanical components, with studies of risk of fatigue damage or line breakage under extreme conditions [10], [11], [8]. Human interactions are still the significant factors to handle instantaneous faults. However, the uncertainty of human interaction is high. A review of the accident databases shows that human error is the dominant factor in maritime accidents [12]. To prevent risk and to reduce costs, faults should be isolated and handled automatically by a fault-tolerant control capability of the position mooring control system. One of the approaches to detect faults is the structural analysis technique [13]. Once a fault has been found in a component of the system, the controller is to be re-designed such that the faulty effect can be attenuated. The control re-configuration strategy may be designed dependent on the type of the fault and thus implemented as needed.

Systematic fault diagnosis and tolerant control for station-keeping of a marine vessel was found in [14]. A structural analysis technique is utilised to generate the residual for the fault diagnosis in [14] and control re-configuration design was applied to alleviate the effects of thruster failure. For sensor failure, such as temporary loss of sensor data, faulttolerant sensor fusion was demonstrated in [15]. These strategies were validated in sea trials. For PM systems, the tension compensation with feedforward control in the case of line breakage was suggested in [4]. The structural analysis was further applied on the PM problem in [16] and an off-line fault-accommodation strategy was designed with the switch between a bank of controllers in the event of mooring line breakage [7]. Systemwide fault-tolerant control of a PM system was demonstrated in [17] where active fault isolation was used to isolate faults that are otherwise only detectable. This fault-handling control was based on thruster usage with the reference position maintained, also when accommodating the effects of line failure. An optimal set-point chasing algorithm was proposed in [18], where it was suggested to move the reference position to an optimal position where a tension-based cost function achieves a minimum value. This paper investigates diagnosis of several mooring system failures, including loss of underwa- ter buoyancy elements, and the paper shows how diagnosis is combined with reliability-based faulttolerant control. The diagnosis principles employed in this paper are based on early results reported in [19] which are further refined and combined into a total fault-tolerant control scheme in this paper.

Reliability of a marine structure was initially combined with a control algorithm by [20] for an offshore drilling platform with a rigid riser. Extension to a moored structure was dealt with in [21], [22] and [23], where an explicit account of external environment was done through a reliability index. For mooring systems, [22] utilised the structural reliability index for off-line calculation of thrust needed. A reliability-based control algorithm was further proposed in [23], making the reliability index an intrinsic part of a controller. This algorithm could handle one critical mooring line - the one with the smallest reliability index. The structural reliability index was further proposed into a set-point chasing algorithm in [11] to reduce the risk of mooring line breakage in an extreme environmental situation and fault-tolerance aspects were also considered. This paper aims to combine the structural reliability index into the fault-accommodation strategy suggested in [18]. In the case of mooring line breakage, the fault-tolerant control helps to prevent the structural reliability index of the mooring line from a critical index.

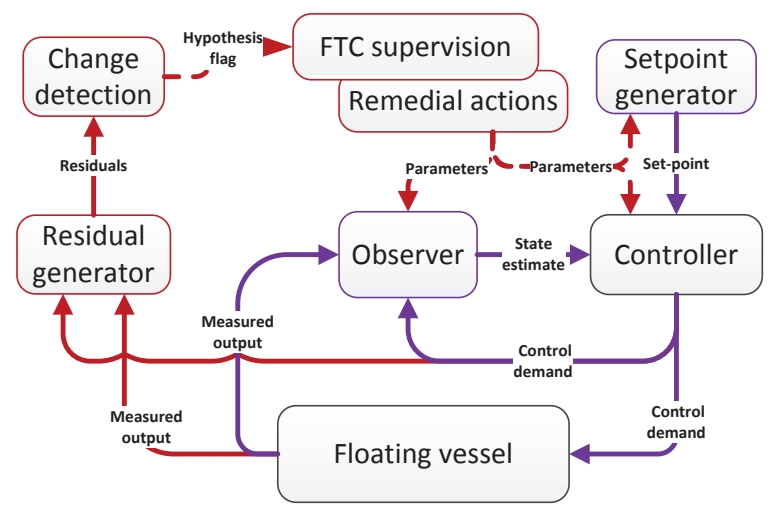

Figure 1: Architecture of fault-tolerant control using setpoint for fault-handling

Fig. (1) shows the fault-tolerant control architecture for the PM system. Once there is a fault in the system, the residual generator diagnoses the fault with the measured signals and control input. Related residuals for a specific fault would be produced accordingly, which are then delivered to the 
change detection algorithm. The change of the residuals is then found by the dedicated detector. The generated hypothesis flags are supervised to decide the remedial actions. If the remedial decision is made, the design of observer and controller is adjusted based on the system model in the faulty case. The set-point chasing algorithm further produces the suitable reference position for the PM system to ensure system safety. This paper concerns the change detection and set-point generation illustrated in Fig. (1).

The paper is structured as follows. Residual generation is first obtained through structural analysis of the nonlinear system. Properties of residuals are then studied and whitening is applied to meet theoretical pre-conditions. Section 3 analyses probability distributions for the experimental data and a change detection algorithm is designed. Fault tolerant control with an optimal set-point chasing algorithm is then described and simulations and experimental results appear in Section 5, showing convincing agreement between model and experiments. Conclusions finish the paper.

\section{Structural analysis}

Modelling of the PM system is defined as the set of the constraints, shown in Appendix A. A typical PM system is shown in Fig. (2). It should be noticed that the numbers of thrusters, sensors and measurement units depend on the class of DP system. Detailed class regulations are found in [24] and [25]. The modelling here presents the normal behaviour of the PM system, and structural analysis is used to find the over-determined subsystem. It finds a complete matching with respect to unknown variables and uses non-matched constraints, if these exist, as the analytical redundancy relations, also known as the parity relations or residual vectors.

SaTool is a software developed for the structural analysis technique. With SaTool, a set of parity relations is generated as the result of structural analysis in symbolic form. Occurrence of a fault is considered as a deviation from the constraints. This deviation will affect a parity relation if this parity relation is built from the constraints. The parity relations can then be used as residual generators for fault detection in the system. Among the analytical redundancy relations that exist for the system, Eqs. (1) - (3) are sensitive to faults on the $i$ th mooring line:

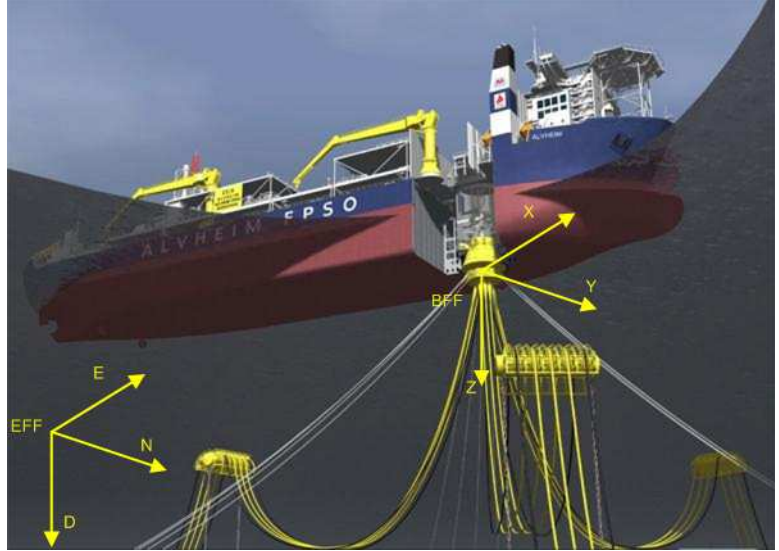

Figure 2: A typical PM system. By courtesy of Berntsen [23]

The analytical redundancy relation $\mathbf{a}_{1}^{r r}$ is a vector expressing $x, y$ linear acceleration balance, $a_{2}^{r r}$ is a scalar with angular acceleration balance and $a_{5+i}^{r r}$ where $i=1,2, . ., 6$ is a vector expressing the balance between estimated and measured tensions in the six mooring lines. For brevity, other residuals, which are not related to mooring lines, are not included here.

The parity relations 4 to 6 below, are found by inserting the constraints listed in Appendix A in the symbolic expressions 1 to 3 and introducing, for brevity, $\mathbf{p}_{\text {rel }} \triangleq \mathbf{p}_{H 1}^{m}-\mathbf{R}\left(\mathbf{q}_{1}\right) \mathbf{l}_{H 1}$, the analytical form of residuals become Eqs. (4)-(6).

In Eqs. (4)-(6), $r_{1}$ and $r_{2}$ are the $x$ and $y$ components of $\mathbf{a}_{1}^{r r}$. The impact on yaw acceleration $a_{2}^{r r}$ is minor for a loss of a buoyancy element or breakage of line $j$, would affect the residual vector components $\left[r_{1}, r_{2}, \ldots, r_{5+j}\right]$.

In Eqs. (4)-(6), $\psi_{1}, \psi_{2}, \psi_{3}$ are the yaw angle measurements, $\psi, \dot{\psi}$ are the yaw angle and yaw rate, $\mathbf{p}_{G 1}, \mathbf{p}_{G 2}, \mathbf{p}_{H 1}$ are the position measurements, $\mathbf{p}, \dot{\mathbf{p}}$ are vessel position and velocity, $\mathbf{q}_{1}, \mathbf{q}_{2}, \mathbf{q}_{3}$ are the vertical reference measurements, $z, \phi, \theta$ are vessel heave, roll and pitch, $\mathbf{w}_{m 1}, \mathbf{w}_{m 2}, \mathbf{c}_{m}$ are wind and current measurements, $\mathbf{v}_{w}, \mathbf{v}_{c}$ are wind and current velocity, $\mathbf{T}_{\text {wave }}$ is the wave force, $\mathbf{T}_{m o j}$ is the mooring line tension, $\mathbf{T}_{m b i}$ is the MLBE force, $\mathbf{T}_{m j}$ is the mooring line tension measurement in line $i, \mathbf{v}$ is the vessel velocity, $\mathbf{v}_{m}$ is velocity measurement, $u_{1}, u_{2}, \ldots u_{k}$ are thruster input, $T_{1}, T_{2}, T_{3}$ are the thruster forces. The detailed modelling for a PM system is described, e.g. in [26]. 


$$
\begin{aligned}
& \mathbf{a}_{1}^{r r}=c_{1}\left(a_{1}\left(u_{1}\right), a_{2}\left(u_{2}\right), c_{6}\left(m_{3}\left(\psi_{3}\right), m_{9}\left(\mathbf{m}_{3}\right), m_{6}\left(\mathbf{p}_{m H 1}\right)\right), m_{3}\left(\psi_{3}\right),\right. \\
& c_{2 i+5}\left(c_{6}\left(m_{3}\left(\psi_{3}\right), m_{9}\left(\mathbf{m}_{3}\right), m_{6}\left(\mathbf{p}_{m H 1}\right)\right), m_{3}\left(\psi_{3}\right)\right. \text {, } \\
& \left.\left.c_{2 i+6}\left(c_{6}\left(m_{3}\left(\psi_{3}\right), m_{9}\left(\mathbf{m}_{3}\right), m_{6}\left(\mathbf{p}_{m H 1}\right)\right), m_{3}\left(\psi_{3}\right)\right)\right), m_{12}\left(\mathbf{w}_{m 2}\right), d_{3}\left(m_{3}\left(\psi_{3}\right)\right)\right), \\
& a_{2}^{r r}=c_{2}\left(a_{3}\left(u_{3}\right), c_{6}\left(m_{3}\left(\psi_{3}\right), m_{9}\left(\mathbf{m}_{3}\right), m_{6}\left(\mathbf{p}_{m H 1}\right)\right), m_{3}\left(\psi_{3}\right),\right. \\
& c_{2 i+5}\left(c_{6}\left(m_{3}\left(\psi_{3}\right), m_{9}\left(\mathbf{m}_{3}\right), m_{6}\left(\mathbf{p}_{m H 1}\right)\right), m_{3}\left(\psi_{3}\right),\right. \\
& \left.\left.c_{2 i+6}\left(c_{6}\left(m_{3}\left(\psi_{3}\right)\right)\right), m_{12}\left(\mathbf{w}_{m 2}\right), d_{3}\left(m_{3}\left(\psi_{3}\right)\right), m_{3}\left(\psi_{3}\right), m_{9}\left(\mathbf{m}_{3}\right), m_{6}\left(\mathbf{p}_{m H 1}\right)\right), d_{4}\left(d_{3}\left(m_{3}\left(\psi_{3}\right)\right)\right)\right) \\
& a_{5+i}^{r r}=m_{13+j}\left(\mathbf{T}_{m j}, c_{2 i+5}\left(c_{6}\left(m_{3}\left(\psi_{3}\right), m_{9}\left(\mathbf{q}_{3}\right), m_{6}\left(\mathbf{p}_{H 1}^{m}\right)\right), m_{3}\left(\psi_{3}\right),\right.\right. \\
& \left.\left.c_{2 i+6}\left(c_{6}\left(m_{3}\left(\psi_{3}\right), m_{9}\left(\mathbf{q}_{3}\right), m_{6}\left(\mathbf{p}_{H 1}^{m}\right)\right), m_{3}\left(\psi_{3}\right)\right)\right)\right) . \\
& {\left[\begin{array}{l}
r_{1} \\
r_{2}
\end{array}\right]=\mathbf{M}^{-1}\left(\mathbf{H}_{x y} \mathbf{T}\left[g_{1}\left(u_{1}, u_{2}, \ldots, u_{k}\right), g_{2}\left(u_{1}, u_{2}, \ldots, u_{k}\right), g_{3}\left(u_{1}, u_{2}, \ldots, u_{k}\right)\right]^{\top}\right)} \\
& +\mathbf{M}^{-1}\left(\left[\begin{array}{ll}
g_{w}^{x}\left(\mathbf{w}_{m 1}\right) & g_{w}^{y}\left(\mathbf{w}_{m 1}\right)
\end{array}\right]^{\top}+\sum_{j=1}^{n} \mathbf{A}_{m o}^{x y}\left(\mathbf{p}_{\mathbf{r e l}}, \psi_{1}\right), \mathbf{T}_{m o j}^{x y}\left(g_{m o}\left(\mathbf{p}_{\text {rel }}, \psi_{1}, g_{m b}\left(\mathbf{p}_{\text {rel }}, \psi_{1}\right)\right)\right)\right) \\
& -\mathbf{M}^{-1}\left(\mathbf{D A}_{v e}^{-1}\left[\frac{\partial}{\partial t}\left(\mathbf{p}_{r e l}-\mathbf{c}_{m}\right) \quad \frac{\partial}{\partial t}\left(\psi_{1}\right)\right]^{\top}\right)-\frac{\partial}{\partial t} \frac{\partial}{\partial t}\left(\mathbf{p}_{\text {rel }}\right) \\
& {\left[r_{3}\right]=\mathbf{I}_{z z}^{-1}\left(\mathbf{H}_{\psi} \mathbf{T}\left[g_{1}\left(u_{1}, u_{2}, \ldots, u_{k}\right), g_{2}\left(u_{1}, u_{2}, \ldots, u_{k}\right), g_{3}\left(u_{1}, u_{2}, \ldots, u_{k}\right)\right]^{\top}+g_{w}^{\psi}\left(\mathbf{w}_{m 1}\right)\right)} \\
& +\mathbf{I}_{z z}^{-1}\left(\sum_{j=1}^{n} \mathbf{A}_{m o}^{\psi}\left(\mathbf{p}_{\text {rel }}, \psi_{1}\right) \mathbf{T}_{m o j}^{\psi}\left(g_{m o}\left(\mathbf{p}_{\text {rel }}, \psi_{1}, g_{m b}\left(\mathbf{p}_{r e l}, \psi_{1}\right)\right)\right)\right) \\
& {\left[\begin{array}{l}
r_{6} \\
\vdots \\
r_{11}
\end{array}\right]=\mathbf{T}_{m}-g_{m o}\left(\mathbf{p}_{\text {rel }}, \psi_{1}, g_{m b}\left(\mathbf{p}_{\text {rel }}, \psi_{1}\right)\right)}
\end{aligned}
$$
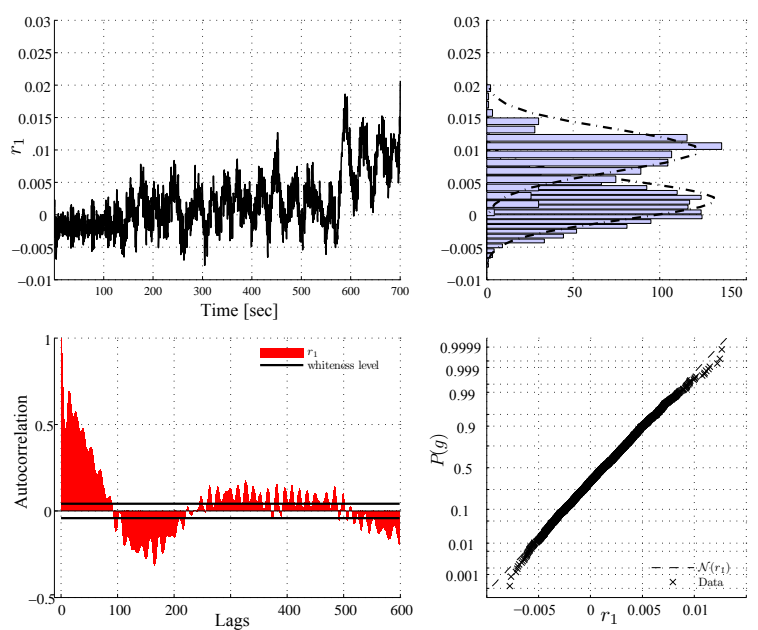

Figure 3: Time history, autocorrelation, PDF and CDF for raw residual $r_{1}$ with loss of buoyancy element at $t=580 \mathrm{~s}$. The PDF is shown before and after loss of the MBLE. A Gaussian probability plot is used for the CDF.

\subsection{Detectability and isolability}

If a fault affects the residual vector, the fault is structurally detectable. If a particular fault has a unique pattern in the residual vector's elements, it is structurally isolable. In the presence of only one fault, isolable constraints are $\left(m_{1}, m_{2}, m_{3}, m_{7}, m_{8}, m_{9}, m_{10}, m_{11}, m_{13+j}\right)$. The rest are detectable. Considering the faults on the mooring line, violations of constraints $c_{2 i+5}$ and $c_{2 i+6}$ are detectable and their residual vectors are unique from those of the other cases. On breakage of a line, the measured tension on $m_{13+j}$ becomes zero. In contrast, with loss of a buoyancy element, the tension will increase. One can hence distinguish between these two faults by detecting the tension change of the measurement. The constraints $m_{13+j}$ are isolable and thus the fault on tension measurement equipment can be distinguished from the fault on the mooring line. 
If two or more faults are present simultaneously, fault isolation, and therefore also fault accommodation, becomes more complex and active fault isolation [27] may be required. This was demonstrated on the PM system in [17].
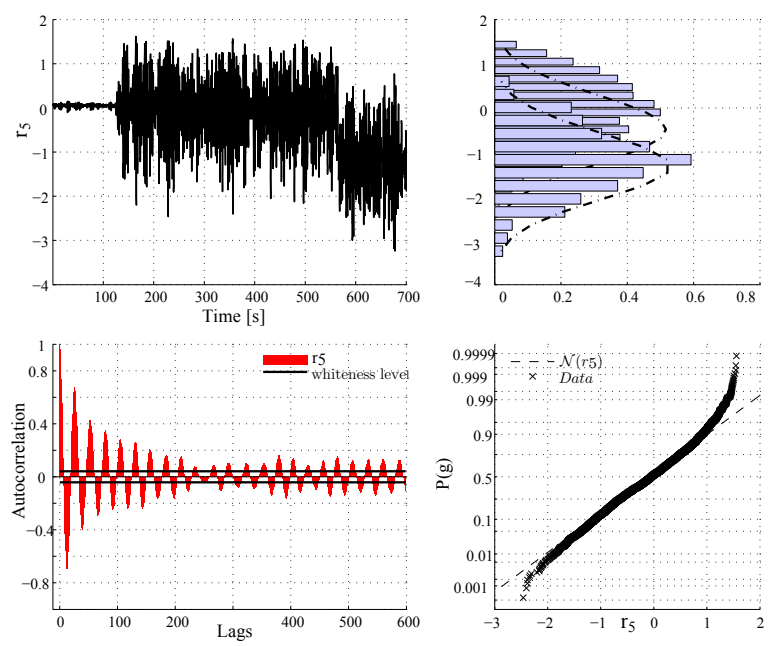

Figure 4: Time history, autocorrelation, $\mathrm{PDF}$ and CDF for raw residual $r_{5}$ with sudden loss of a buoyancy element (MBLE) at $t=580 \mathrm{~s}$. The PDF plot shows histograms of probability density before and after loss of the MBLE. The $\mathrm{CDF}$ is the one before the fault $\left(\mathcal{H}_{0}\right)$ plotted in a Gaussian probability plot.

\subsection{Properties of residuals}

The time-histories, autocorrelation and distributions of the residuals $r_{1}$ and $r_{5+i}$ before and after a fault are shown in Figures 3 and 4 . A similar analysis of residual $r_{2}$ was also made. In the experiment, the wave-making starts at $t=150 \mathrm{~s}$ and a buoyancy element loss at line no.4 is injected at time $t=580 \mathrm{~s}$. Vessel data and experiment details are described in Section 5.1. The auto-correlation functions for $r_{1}$ and $r_{5}$ for the no-fault condition $\left(\mathcal{H}_{0}\right)$ and the PDFs $p\left(r, \mathcal{H}_{0}\right)$ are calculated for the interval $[180,550] s$. The amplitude distributions before and after the fault are reasonably close to being Gaussian. The autocorrelation functions show considerable correlation, caused by the wave impact. This is undesired for change detection since the theory requires independent increments of the residuals (whiteness). Signal whitening is therefore required.

\subsection{Whitening of residuals}

If the discrete time scalar residual $r_{i}\left(k T_{s}\right)$ was generated through an auto regressive moving aver- age (ARMA) model by a scalar process $e\left(k T_{s}\right)$ with independent increments, where $k$ is increments in time and $T_{s}$ the sampling time,

$$
r_{i}\left(k T_{s}\right)=\frac{C(q)}{F(q)} e\left(k T_{s}\right)
$$

where $F(q)=a_{1} q^{1}+a_{2} q^{2}+\cdots+a_{n} q^{n}$, and $C(q)=c_{1} q^{1}+a_{2} q^{2}+\cdots+a_{n} q^{m}$ are polynomials in the delay operator $q$, then, provided $C(q)$ is Hurwitz, the original residual, $r_{i}(k)$ for brevity, would be whitened to $r_{i}^{w}(k)$ by

$$
r_{i}^{w}(k)=\frac{F(q)}{C(q)} r_{i}(k)
$$

Standard identification methods can be used to determine the polynomials in the ARMA model Eq. (7). However, $C(q)$ need be restricted to have eigenvalues within the unit circle. The orders of $F(q)$ and $B(q)$ are determined as part of the identification. The main issue in this procedure is that a model as Eq. (7) requires $e(k)$ to be stationary, but waves of a short-crested sea are not stationary. Furthermore, there are non-linear phenomena involved in forming the mooring line tensions, so a linear whitening process will not suffice. Therefore, the whitening can at best be approximate.

When applying the whitening procedure, the distribution of the raw whitened residual, shown in Fig. (6) appears to have a mixed distribution where the bulk of samples are Gaussian but the tails are differently distributed; the tails have some resemblance to outliers. One approach would be to attempt to fit a distribution to the $\mathcal{H}_{0}$ data and develop dedicated detection. Another is to borrow an idea from robust statistics [28], where a nonlinear transformation, a Huber compression, is applied to data, $r_{i}^{w}$ to give $r_{i}^{w l}$

$$
\begin{aligned}
r_{i}^{w l}(k) & =r_{i}^{w}(k) \frac{1}{1+\gamma(k)} \\
\gamma(k) & =\left|\frac{r_{i}^{w}(k)}{\overline{r_{i}^{w}}}\right|
\end{aligned}
$$

where

$$
\overline{r_{i}^{w}}=k_{\sigma} \sigma\left(r_{i}^{w l}\right)
$$

is an iterative procedure to determine the standard deviation of $r_{i}^{w l}$ from a window of antecedent data. The factor of $k_{\sigma}$ is an engineering design choice, determining the level where the tail of the distribution should be compressed, here $k_{\sigma}=3$ was selected. 
Results of the whitening and subsequent Huber compression are shown in Figs. (5) and (6). It is noted that the accepted theoretical procedure is to first determine a nonlinear function that brings the distribution back to Gaussian and then to whiten the signals. In this case, the raw residuals appeared to be Gaussian but not white, and the nonlinear transformation was applied after whitening. The autocorrelation function of the compressed residuals were found to be reasonably independent, as shown in Fig. (7)
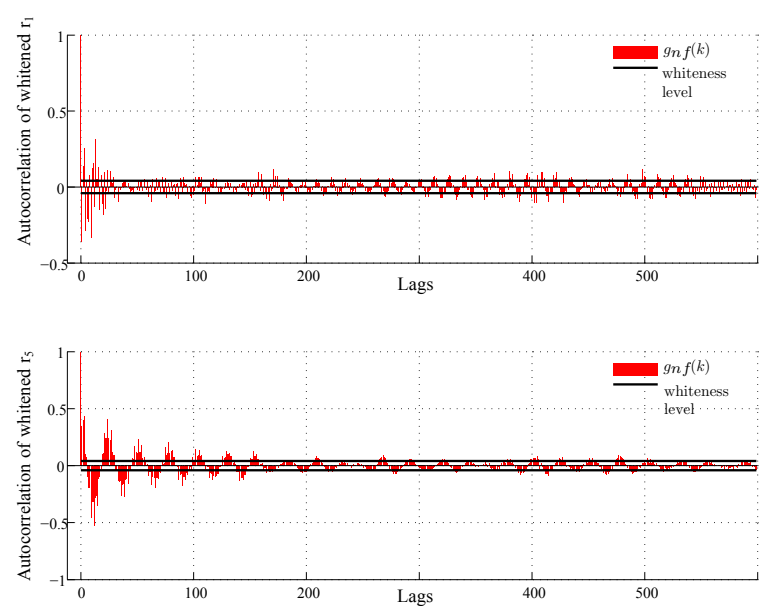

Figure 5: Autocorrelation of whitened residuals $r_{1}$ and $r_{5}$ in a fault-free situation

In Fig. (5), the correlation of residuals $r_{1}$ and $r_{5}$ in a fault-free situation reduces after the whitening process, compared with the residual without a whitening process, shown in Fig. (3) and (4). However, the whitened signals do not approximate the Gaussian distribution. From Fig. (6), the upper and lower tails of the residual CDF (blue point line) are not confirmed very well with the Gaussian distribution.

Fig. (6) and (7) also shows the effect of applying the Huber compression. In Fig. (6), the whitened signals are shown as the blue line, while the result of the compression is shown in green. It is clear that the result of compression is a better fit to Gaussian distribution, which is a straight line in the probability plot. Fig. (7) shows the autocorrelation function of the signals with Huber compression, implying that signal correlation has not changed. Even though the autocorrelation does not show independent increments of the residual, this is the best result that could be achieved by using a fixed
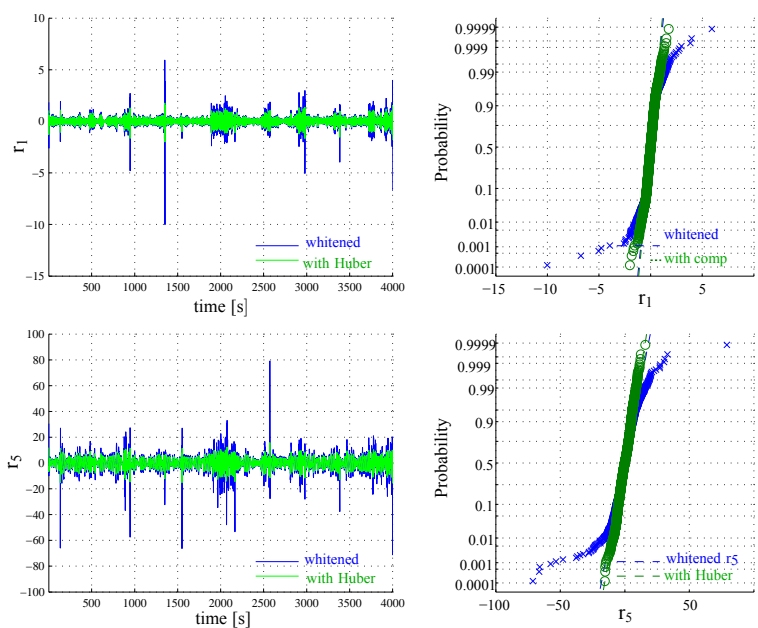

Figure 6: Time histories and cumulative probability distributions of residuals $r_{1}^{w}$ and $r_{5}^{w}$ before and after Huber Compression in condition $\mathcal{H}_{0}$
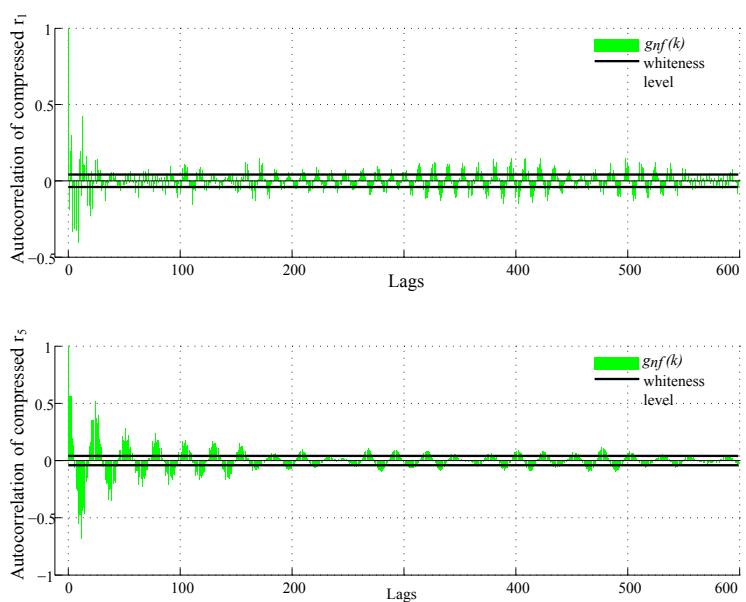

Figure 7: Autocorrelation of compressed residuals $r_{1}^{w}$ and $r_{5}^{w}$ for condition $\mathcal{H}_{0}$

whitening filter on the the non-stationary signal at hand. The change in residuals with loss of buoyancy element are by far the smaller compared to changes appearing when line breakage takes place. Both phenomena are strongly detectable in the residuals Eqs. (4)-(6). This is confirmed by the model tests, shown in Figs. (3) and (4).

From the above observations, the following procedure is adopted for analysis of residuals: residuals are first compressed, thereafter whitening is applied to each component and finally change detection is applied. The residual can be assumed to have a 
Gaussian distribution before and after the change, and the detection aims to find a shift in mean value.

\section{Change detection}

Having established a residual vector with desired properties (Gaussian and independent samples), change detection is a standard problem from detection theory $[29,30]$. The result is repeated here for convenience.

The vector-based hypothesis test problem is formulated as:

$$
\begin{aligned}
\mathcal{H}_{0}: & \mathbf{z}(i)=\boldsymbol{\mu}_{0}+\mathbf{w}(i) & i=1, \ldots, k \\
\mathcal{H}_{1}: & \mathbf{z}(i)=\boldsymbol{\mu}_{1}+\mathbf{w}(i) & i=0, \ldots, k,
\end{aligned}
$$

where $\mathbf{w}(i)$ is a Gaussian-distributed vector with independent increments and variance $\mathbf{Q}$, and $\boldsymbol{\mu}_{0}$ and $\boldsymbol{\mu}_{1}$ are mean value vectors before and after the change, respectively. A special case, which applies here, is that the direction of change is known but the magnitude of change is unknown. Let $\boldsymbol{\Gamma}$ be the known direction of change, and $v$ an unknown scalar, then

$$
\mathcal{H}_{1}: \quad \boldsymbol{\mu}_{1}=\boldsymbol{\mu}_{0}+\boldsymbol{\Gamma} v .
$$

The generalised likelihood ratio test (GLRT) on a sequence of $k$ samples will decide $\mathcal{H}_{1}$ if

$$
L_{G}=\sum_{i=1}^{k} \frac{p\left(\mathbf{z}(i) ; \hat{v}, \mathcal{H}_{1}\right)}{p\left(\mathbf{z}(i) ; \mathcal{H}_{0}\right)}>\gamma_{0} ;
$$

where $\hat{v}_{1}$ is the maximum likelihood estimate (MLE) of $\boldsymbol{\mu}_{1}$, and $\gamma_{0}$ is a threshold selected to give a desired (low) probability of false alarms.

The MLE of $v$ is found where the gradient of $\ln L_{G}$ is zero and as

$$
\frac{\partial \ln L_{G}}{\partial v}=\boldsymbol{\Gamma}^{\prime} \mathbf{Q}^{-1} \sum_{i=1}^{k}\left(\mathbf{z}(i)-\boldsymbol{\mu}_{0}\right)-k \boldsymbol{\Gamma}^{\prime} \mathbf{Q}^{-1} \boldsymbol{\Gamma} v,
$$

the MLE estimate of $v$ is,

$$
\hat{v}=\frac{\boldsymbol{\Gamma}^{\prime} \mathbf{Q}^{-1} \frac{1}{k}\left(\sum_{i=1}^{k} \mathbf{z}(i)-\boldsymbol{\mu}_{0}\right)}{\boldsymbol{\Gamma}^{\prime} \mathbf{Q}^{-1} \boldsymbol{\Gamma}} .
$$

Using $g=\ln \left(L_{G}\right)$ as test statistic, the explicit form of the detector is, see [13],

$$
\begin{aligned}
& g(k)= \\
& \quad\left(\boldsymbol{\Gamma}^{\prime} \mathbf{Q}^{-1} \frac{1}{k} \sum_{i=1}^{k}\left(\mathbf{z}(i)-\boldsymbol{\mu}_{0}\right)\right)^{2}\left(\boldsymbol{\Gamma}^{\prime} \mathbf{Q}^{-1} \boldsymbol{\Gamma}\right)^{-1}
\end{aligned}
$$

If at the sample $k$ the $g(k)$ is larger than the chosen threshold $\gamma$, where $\gamma=\ln \left(\gamma_{0}\right)$ above, then the hypothesis $H_{1}$ is accepted.

In an on-line implementation, a moving window of the latest $M$ samples is considered and the test statistic for line $j$ is, using $\boldsymbol{\Gamma}_{j}$ for the known direction caused by fault on this line,

$$
\begin{aligned}
& g_{j}(k)= \\
& \quad\left(\boldsymbol{\Gamma}_{j}^{\prime} \mathbf{Q}^{-1} \frac{1}{M} \sum_{i=k-M}^{k}\left(\mathbf{z}(i)-\mu_{0}\right)\right)^{2}\left(\boldsymbol{\Gamma}_{j}^{\prime} \mathbf{Q}^{-1} \boldsymbol{\Gamma}_{j}\right)^{-1}
\end{aligned}
$$

and the estimate of the magnitude of change is

$$
\hat{v}_{j}=\frac{\boldsymbol{\Gamma}_{j}^{\prime} \mathbf{Q}^{-1} \frac{1}{M}\left(\sum_{i=k-M}^{k} \mathbf{z}(i)-\boldsymbol{\mu}_{0}\right)}{\boldsymbol{\Gamma}_{j}^{\prime} \mathbf{Q}^{-1} \boldsymbol{\Gamma}_{j}} .
$$

The detector in Eqs. (15)-(16) are used in the sequel for the processing of experiment data.

\subsection{Alternative change detection schemes}

CUSUM based change detection is efficient in detecting a change of known magnitude and the CUSUM approach was employed in [18] on the position mooring application, and it was shown to give very convincing results for the known magnitude events, which are: loss of a buoy, or a line breakage. This paper uses the vector based GLR test, as the GLR has the ability to detect a change of unknown magnitude. This implies that also partial loss of buoyancy can be detected.

Fault isolation is achieved in the vector-based approach through the known direction of change for each particular fault. Since a fault in mooring line $j$ will manifest itself in the vector $\mathbf{r}=\left[r_{1}, r_{2}, \ldots r_{5+j}\right]^{\prime}$. The directions of change $\boldsymbol{\Gamma}_{j}$ differ in one component and isolation could be achieved in two ways.

One way could be to model the change as

$$
\mathcal{H}_{\infty}: \quad \mathbf{z}_{i}=\boldsymbol{\mu}_{1}+\boldsymbol{\mu}_{0}+\mathbf{w}_{i}
$$

and consider $\boldsymbol{\mu}_{1}$ as the unknown parameter to be estimated within the GLR. Isolation would then be achieved by projection of the estimate $\hat{\boldsymbol{\mu}}_{1}$ on each of the possible failure directions $\boldsymbol{\Gamma}_{j}$ and finding the index $j_{f}$ of the faulty line as,

$$
j_{f}=\max _{j \in n}\left(\hat{\boldsymbol{\mu}}_{1} \cdot \boldsymbol{\Gamma}_{j}\right)
$$


The approach used here is to run parallel vector detectors Eqs. (16) and (15) and isolate by

$$
j_{f}=\max _{j \in n}\left\{g_{j}(k) \mid g_{j}(k)>\gamma\right\}
$$

The two vector-based methods have fairly similar performance in this application.

\subsection{Threshold determination}

In theory, asymptotic analysis of the GLRT detector, i.e. analysis of the distribution of the test statistics $g(k)$, could provide the threshold $\gamma$ that would give a certain false alarm probability [30]. However, with a limited size of samples in the GLRT, asymptotic results are not accurate and the empirical distribution of $g(k)$ need be examined.

Selection of $\gamma$ to obtain a sufficiently low falsealarm rate $P_{F A}$, depends on the statistics of $g(k)$ under the assumption $\mathcal{H}_{0}$. Fig. (8) shows $g(k)$ for the vector residual. The information from three signals is combined to indicate the loss of buoyancy element. It is shown that at $t=580$, the test statistics is changed sharply. The red line shows the threshold that triggers an alarm to indicate the loss of a buoyancy element. The asymptotic distribution of GLRT statistics at $\mathcal{H}_{0}$ is then selected to calculate the threshold for the detector.
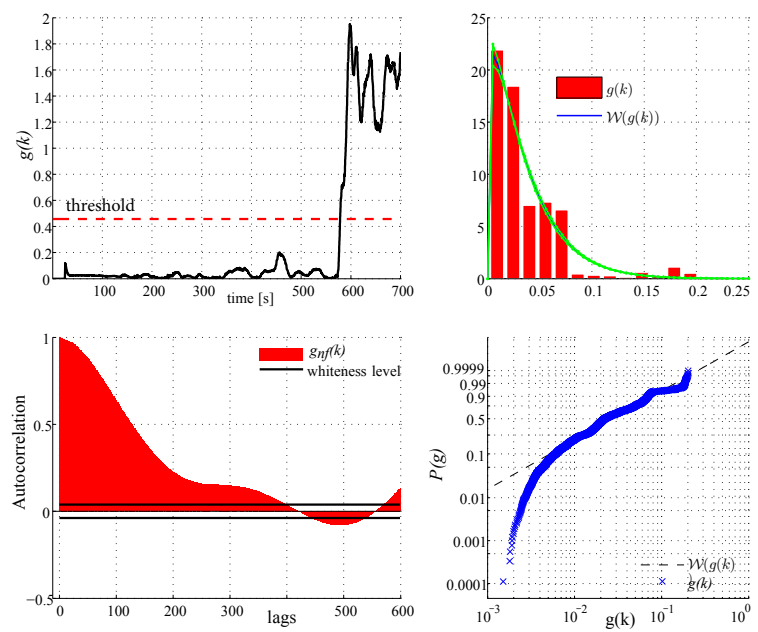

Figure 8: Features of test statistics $g_{4}(k)$ for the vector residual: time history, histogram and approximating PDF, autocorrelation and CDF plotted as a Gaussian probability plot.

Fig. (8) shows the autocorrelation function for the test statistics $\mathrm{g}(\mathrm{k})$ for the vector-based GLRT detector using a window length of 400 samples. The autocorrelation is calculated from data with no fault injected, when the $\mathcal{H}_{0}$ assumption is valid. The test statistics are clearly correlated. It is evident that the theoretical IID assumption for the GLRT statistics is not fulfilled. Hence the distribution of the test statistics for the no-fault condition $\left(\mathcal{H}_{0}\right)$ will needs to be learned from data in order to find a threshold that gives a desired (low) probability for false alarms.

The distribution of the GLRT test statistics is approximated quite well with a Weibull distribution. The PDF and CDF of data and the approximating distribution are shown in Fig. (8). The vector residual is approximately Gaussian, which is to be expected when the residuals are dominated by wave frequency vessel motions. Second order, slowly varying, wave forces exist and create nonlinearity in the vessel motions, but this was not a dominating component in the experiments. The statistics of a GLRT on Gaussian signals exist as theoretical, asymptotic expressions [30], with a window size reasonable for implementation (window size is 150 samples). Admittedly, the building of the GLRT statistic itself is also a nonlinearity process.

The threshold $\gamma$ is selected to obtain a sufficiently low false-alarm rate $P_{F A}$ with the Weibull distribution of the GLRT statistic as:

$$
P_{F A}=\int_{g: L_{G}(\mathbf{z}(k))>\gamma} p_{W}\left(g ; \mathcal{H}_{0}\right) d g ;
$$

where $p_{W}\left(g ; \mathcal{H}_{0}\right)$ is the PDF of the Weibull distribution for the GLRT statistics under $\mathcal{H}_{0}$.

As shown in Fig. (8), the estimation of the Weibull parameters $v_{0 g}$ and $\beta_{0 g}$ is obtained under $\mathcal{H}_{0}$ as $p_{W}\left(g ; \mathcal{H}_{0}\right)$. The threshold for a desired (low) false alarm probability $P_{F A}$ is then obtained from,

$$
1-P_{F A}=1-\exp \left(-\left(\frac{\gamma}{v_{o g}}\right)^{\beta_{0 g}}\right)
$$

Subsequently, the threshold $\gamma$ is determined from the desired false alarm probability as,

$$
\gamma=v_{0 g}\left(-\ln P_{F A}\right)^{\frac{1}{\beta_{0 g}}}
$$

The false alarm probability is set to a very low value and the threshold is calculated threshold from 22. The threshold is shown as the dotted line in Fig. (8). After the fault on the mooring line is detected, the fault-tolerant strategy is then activated. The target of fault-tolerant control here is to prevent the structural index of the other mooring lines from a critical value after one line breakage or loss of a buoyancy element. 


\subsection{Physical failure mechanisms}

Regarding degradation and subsequent breakage of a mooring line, fatigue or corrosion damage are typical causes for breakage. As mooring lines are most often heavy chains, line breakage occurs due to fatigue of one chain element combined with instantaneously high stress in adverse weather conditions. Breakage is therefore essentially an abrupt event that is hardly visible in the mooring system dynamics before the event. Gradual loss of buoyancy of a buoyancy element is, by contrast, an incipient mechanism.

The breakage of a line or sudden loss of a buoyancy element gives rise to a fairly abrupt change in residuals with a shift in mean value as a consequence. If a leakage causes a buoyancy element to gradually lose buoyancy, this will be reflected in a change in residuals that start departing from zero mean. When filled to neutral buoyancy, the residuals take equivalent values as for the loss of buoy. When completely water filled, the dead weight of the buoy enters into the catenary equation.

\subsection{Detection behaviour for incipient faults}

Assuming a leakage develops linearly, it starts at sample $i_{0}$ and neutral buoyancy is reached after $m$ samples. Then the residual would develop as follows, while $i_{0} \leq i \leq i_{0}+m$,

$$
\mathcal{H}_{\text {leak }}: \quad \mathbf{z}(i)=\mu_{0}+\Gamma \frac{\left(i-i_{0}\right)}{m} \nu+\mathbf{w}(i)
$$

A specific test could be developed for this particular case, but a real leak could develop other than linearly over time. Therefore, it is interesting to investigate how the detector developed above for the abrupt fault will behave in the case of an incipient loss of buoyancy. Assuming $m \gg M$, otherwise detection behaviour would resemble that of the abrupt change, then, while $i_{0}+M<k<i_{0}+m$,

$$
\begin{aligned}
& g(k)= \\
& \left(\boldsymbol{\Gamma}^{\prime} \mathbf{Q}^{-1} \frac{1}{M} \sum_{i=k-M}^{k}\left(\Gamma \frac{\left(i-i_{0}\right)}{m} \nu+\mathbf{w}(i)\right)\right)^{2} \boldsymbol{\Gamma}^{\prime} \mathbf{Q}^{-1} \boldsymbol{\Gamma}
\end{aligned}
$$

The distribution of $g(k)$ under $\mathcal{H}_{\text {leak }}$ will therefore develop from that under $\mathcal{H}_{0}$ to that for $\mathcal{H}_{1}$ when $\frac{\left(i-i_{0}\right)}{m}=1$ by which time buoyancy is neutral. The detector Eq. (15) will give a series of alerts until the leak is sufficiently large to trigger a permanent alarm. Using the magnitude of the change
Eq. (16), the change in physical buoyancy follows from the equations of motion, see Appendix A, from which the change in buoyancy could be calculated and used for real-time warning.

A simpler solution in practice could be to base warning / alarm logic on the estimated magnitude of change since an incipient fault will manifest itself as a gradual change towards a full loss of a buoyancy element, and beyond as it eventually becomes completely filled with water. Hypothesis testing combining model-based FDI with statistical change detection and testing with methods from artificial intelligence could be employed to avoid nuisance alarms on-board. Such methods were covered in the survey by [31] and a study of data driven diagnosis showed design for isolability of different types of faults in automotive engines [32]. The same authors suggested an explicit comparison of the probability distribution of the residual, estimated online using current data, with no-fault residual distributions in [33] and found that tiny faults, i.e. also incipient ones, could be diagnosed in conditions where traditional methods would fall short.

\section{Fault-tolerant control}

This section will summarize the properties of the fault-tolerant set-point-chasing control algorithm, first introduced in [18] and its properties will be investigated and documented from model tests where faults were imposed.

The set-point chasing algorithm recalculates an optimal set-point for the mooring system, within an allowed range, to protect the mooring lines from breakage or, if one or more lines have failed, calculate the optimal set-point that protects all remaining lines. Weather conditions and mooring system dynamics are incorporated through estimation of the distribution of line tensions and calculation of an associated risk factor, [11].

\subsection{Controller design}

The control objective is to maintain the vessel's position in a limited region and keep the vessel on the desired heading such that the external environmental load is minimised. Another objective is to avoid line breakage and keep the mooring system in a safe state. An optimal position algorithm is designed to meet the second objective. The control architecture for the PM system is shown in Fig. (1).

The control action for the PM also aims to counteract the low-frequency part of vessel motion 
caused by current and wind as well as second order mean and slowly varying wave loads. The specific function of control action in PM can be found in [34]. For the controller design, it is common to use multi-variable PID control in PM systems with the structure:

$$
\begin{aligned}
\boldsymbol{\tau}_{t h r}= & -\mathbf{K}_{d} \hat{\boldsymbol{\nu}}_{e}-\mathbf{K}_{p} \mathbf{R}^{T}(\psi) \hat{\boldsymbol{\eta}}_{e} \\
& -\mathbf{K}_{i} \mathbf{R}^{T}(\psi) \int \hat{\boldsymbol{\eta}}_{e} d t
\end{aligned}
$$

where $\hat{\boldsymbol{\eta}}_{e}=\hat{\boldsymbol{\eta}}-\boldsymbol{\eta}_{d}$ and $\hat{\boldsymbol{\nu}}_{e}=\hat{\boldsymbol{\nu}}-\boldsymbol{\nu}_{d}$ are the position and velocity errors; $\boldsymbol{\eta}_{d}$ and $\boldsymbol{\nu}_{d}$ the desired position and velocity vectors; and $\mathbf{K}_{d}, \mathbf{K}_{i}$ and $\mathbf{K}_{d} \in \mathbb{R}^{3 \times 3}$ are the non-negative controller gain matrices. $\psi$ is the measured heading angle and $\mathbf{R}(\psi)$ is the rotation matrix from the body-fixed to the Earth-fixed frames. For the detailed design of the controller and observer, refer to Appendix B.

\subsection{Set-point chasing algorithms}

To prevent failure of mooring lines in PM systems, position-moored vessels are kept within a small region, which is normally defined as a circle with a specific radius. This radius is one of the critical factors in order to ensure mooring-line safety [35] [36]. A reliability index has been used to evaluate this region for moored, interconnected structures [22] and a reliability-index-based controller has been designed to maintain the safety of one critical mooring line [23]. The present paper extends previous results by diagnosis and testing of a strategy for fault tolerance against mooring system failures.

\subsubsection{Structural reliability}

The basic idea of the fault-tolerant control on PM systems is to make sure that the probability of mooring-line failure is kept below a predefined acceptable level. The failure probability for a mooring line is expressed in terms of a reliability index, the so-called $\delta$-index, which is described in classical texts, see e.g. [37]. Control schemes based on a reliability index were investigated for risers and mooring systems in [20] and [22]. The index quantifies the probability of mooring-line failure and can be expressed as :

$$
\delta_{i}(t)=\frac{T_{c i}-T_{e x i}}{\sigma_{c i}} \quad i=1, \ldots n
$$

where $T_{c i}$ is the critical strength of the $i$ th mooring line, $T_{\text {exi }}$ is the extreme value of the mooringline tension (also including the slowly-varying mean value). The extreme value of the tension associated with the wave frequency (first order) response is in the present study evaluated over a 30 minutes window, based on $1 \mathrm{~s}$ samples. The standard deviation of the critical strength is $\sigma_{c i}$. In practice, $\sigma_{c i}$ should be based on tests, or alternatively obtained from data sheets provided by the manufacturer. Specifying a permissible lower bound (i.e. critical value) for the reliability index $\delta_{i}$, further denoted by $\delta_{c i}$, also implies the condition $\delta_{i}<\delta_{c i}$ represents a situation where the probability of line failure becomes intolerably high. The fault-tolerant control aims to avoid that this index becomes lower than the critical value.

The index shown in Eq. (26) expresses the risk of breakage caused by extreme tension in a mooring line. With a maximum allowed failure probability, the corresponding extreme value of the tension can be calculated from the cumulative distribution of the extreme value. A procedure for on-line estimation of the extreme value was discussed in [11].

Around a working point, mooring-line tension $T_{i}$ can be expressed as a function of the distance, $\Delta r$, and direction, $\beta$, to a mooring system neutral point where all tensions are equal in calm weather, see [4] and [18]:

$$
\begin{aligned}
T_{i} & =T_{o i}+c_{i} \Delta h \\
& =T_{o i}-c_{i} \Delta r \cos \left(90^{\circ}-\beta-\beta_{i o}\right) \\
& =T_{o i}-c_{i} \Delta r \sin \left(\beta+\beta_{i o}\right) .
\end{aligned}
$$

Here $T_{o i}$ is the tension at the working point $\left(x_{o}, y_{o}\right)$, and $c_{i}$ is the incremental stiffness tension at the present working point.

\subsubsection{Optimal position-chasing}

Based on the structural reliability indices, an object function covering all mooring lines is:

$$
L=\sum_{i=1}^{n} w_{i}\left(\delta_{c i}-\delta_{i}\right)^{2},
$$

where $\delta_{c i}$ is the critical value of the reliability index for line $i$ and $\delta_{i}$ is an estimate of the instantaneous reliability index for the line, $w_{i}$ is the weighting for each line.

Solving the equations where the partial derivative of Eq. (28) with respect to the optimal increment of the vessel position and its direction are set 
to zero, identifies the minimum value of the object function. The optimal increment of vessel position and direction are [18]:

$$
\begin{aligned}
\Delta r & =\frac{K_{11} \sin \beta+K_{12} \cos \beta}{K_{21} \sin ^{2} \beta+K_{b}+K_{23} \cos ^{2} \beta} \\
K_{b} & =2 K_{22} \sin \beta \cos \beta \\
\beta & =\operatorname{tg}^{-1} \frac{K_{11} K_{23}-K_{12} K_{22}}{K_{21} K_{12}-K_{11} K_{22}}
\end{aligned}
$$

where the calculations of the parameters $K_{11}$ $K_{23}$ are shown in the Appendix C. Finally, the updated vessel position and heading becomes:

$$
\boldsymbol{\eta}=\boldsymbol{\eta}_{o}+\Delta r\left[\begin{array}{lll}
\cos \beta & \sin \beta & 0
\end{array}\right]^{\top}
$$

The quadratic object function of Eq. (28) is particularly convenient because an analytical optimum exists. Other functions than quadratic could be used, if this was desired, and the resulting set-point would then be achieved by numerical methods for most object functions.

Through the optimization, the set-point chasing algorithm uses physical properties of the passive mooring lines and will not use excessive thruster power to maintain an equilibrium point that is not natural after a fault has occurred. The set-point chasing algorithm achieves a balance between the new natural equilibrium, where risks for all remaining mooring lines are kept within the safety limits and the need of having the position reference within a specified area, if both aims are physically possible.

\section{Simulation and experimental results}

\subsection{Overview of simulation and experiment}

The simulations were carried out using the Marine Systems Simulator (MSS) [38] developed at Marine Cybernetics Group, NTNU. The MSS is the platform developed in the Matlab/Simulink environment for simulating the moored vessel motion and for implementing the fault-tolerant control strategy in Section 5. The model of CyberShip3 (CS3) is selected for both the experiment and simulation. Six mooring lines are added in the turret of this vessel. The vessel is shown in Fig. (9). The main characteristics are shown in Table 1 . The experiments were performed at the MCLab, which is a 6.45 by $40 m$ test basin specifically designed for testing control strategies on marine vessels.

The simulation was carried out using a JONSWAP model for sea-state energy distribution. The
Table 1: Main characteristics of CS3

\begin{tabular}{lcc}
\hline & Model & Unit \\
\hline length overall & 2.275 & {$[\mathrm{~m}]$} \\
length between particulars & 1.974 & {$[\mathrm{~m}]$} \\
breadth & 0.437 & {$[\mathrm{~m}]$} \\
draught & 0.153 & {$[\mathrm{~m}]$} \\
weight & 74.2 & {$[\mathrm{~kg}]$} \\
azimuth thrusters (three) & 27 & {$[\mathrm{~W}]$} \\
tunnel thruster & 27 & {$[\mathrm{~W}]$} \\
\hline
\end{tabular}

significant wave height $H_{s}$ was $0.06 \mathrm{~m}$ equivalent to $2.2 m$ in full scale, which was the maximum irregular sea the wave maker could provide, and the wave peak period was $0.15 \mathrm{~s}$ in model scale. Current increased from 0 to $0.5 \mathrm{~m} / \mathrm{s}$ (full scale equivalent) from time 370 to $520 \mathrm{~s}$ and the wind speed is equivalent to $8 \mathrm{~m} / \mathrm{s}$ in full scale. It is noticed that the loads from the current and wind in the experiment is modelled by a pulley system shown in Fig. (9(c)).

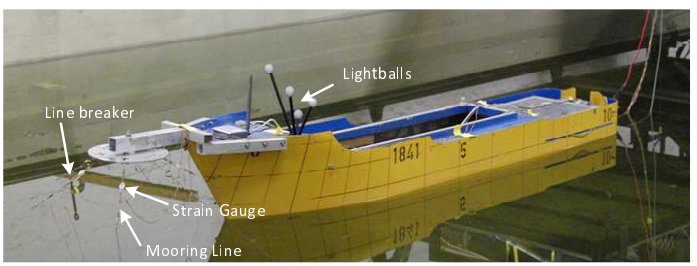

(a) CyberShip III (CS3)
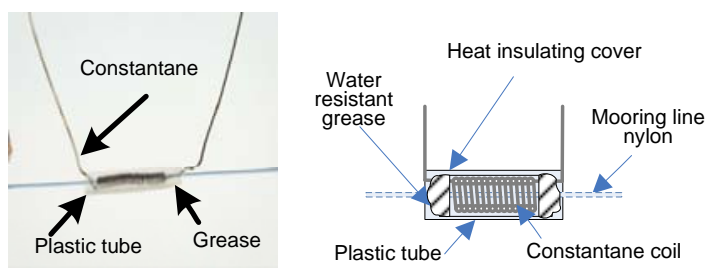

(b) Line breaker

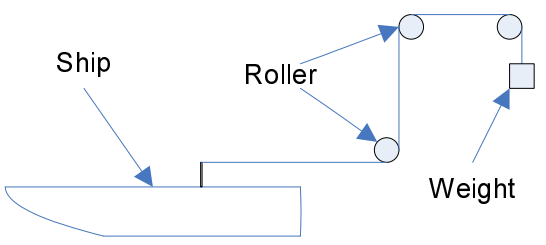

(c) Pulley system

Figure 9: CyberShip III with pulley system

The dimensions of CyberShip3 (CS3) are listed in Table 1 and the experimental set-up is shown in Fig. (9). In front of the bow of vessel, six lines are connected to the turret and a line breaker, shown 


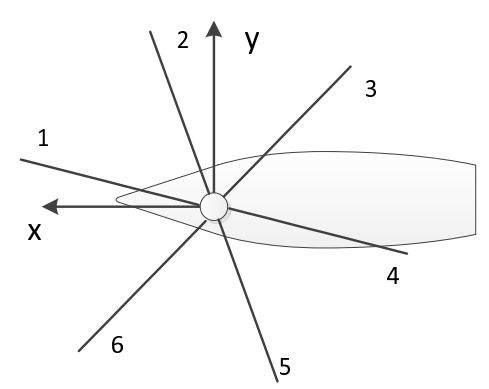

Figure 10: Mooring line position setting

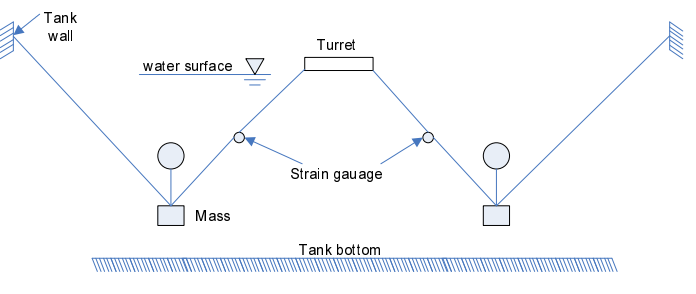

Figure 11: Mooring line arrangement in experiment

in Fig. ( $9(\mathrm{~b}))$, is attached to each line. Fig. ( 10) illustrates the mooring system arrangement. The mooring system model in the experiment was similar to that of [39] shown in Fig. (11). A pulley system shown in Fig. (9(c)) was used to simulate the effect of mean loads due to the wind and current in the experiments.

As shown in Figs. (9) and (11), six cables were connected to the vessel through the turret at the bow to simulate the effect of the catenary system. With this setting, it is convenient to replace the line after one line is broken. Each cable has one end fixed to the turret and the other to the wall of the basin; a mass was suspended between these two points. Strain gauges were attached to the lines to measure the tensions during the experiment. The

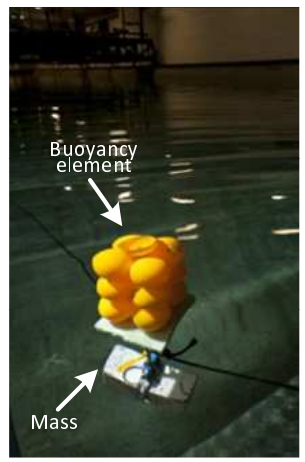

Figure 12: Mooring line buoyancy element measurement of the line force was used to calculate the reliability index. The alloy constantane was used for the heating element, giving the coil a resistance of $0.4 \Omega$. Applying a current of $2 A$ to the element melted the $0.6 \mathrm{~mm}^{\circ}$ nylon line, which passes through the coil, in about one $s$ when submerged under water. Water-proof grease prevented water from entering the interior of the coil and an outer rubber cover provided some heat insulation between the heating element and water.

\subsection{Fault-tolerant control with loss of buoyancy element}

The vessel and mooring system performance with the fault-tolerant control strategy is simulated with the case that the no. 4 line loses a buoyancy element. The simulation result of the loss of buoyancy element is shown in Fig. (13). The experiment for this case needs more efforts. Vessel position with simulation is shown in Fig. (13(a)). Structural reliability indices are shown in Fig. (13(b)). Fig. (13(c)) shows the thruster RPM. Only two thrusters are used here.

The loss of buoyancy element in line no. 4 happens at time $t=700 \mathrm{~s}$. It is shown that line no.1 is already in danger of line failure: a index of less than the critical value $\delta_{c}=4$ and loss of a buoyancy element may make it worse. The index of line no. 6 almost comes into the critical value. These two lines are the ones heavily loaded and both of these should be protected from risk of failure. After the detection of the failure event by the vector detector, the structural-reliability-based fault-tolerant control is activated. It is shown that the index of line no. 1 is then kept beyond the critical value while the indices of the other lines are far from the critical value. Notice that loss of buoyancy element in line no. 4 increases the line tension, which reduces its index.

\subsection{Fault-tolerant control with line breakage}

This section compares the vessel and mooring system performance with the fault-tolerant control strategy in simulation and experiment. The line breakage result is shown in Fig. (14). Vessel position in the simulation and experiment are shown in Figs. (14(a)) and (14(b)). Structural reliability indices and thruster RPM are shown in Figs. (14(c)), (14(d)), (14(e)) and (14(f)).

The overall comparison shows that vessel motion responses are very similar. However, the thruster activity is more fluctuating in the experiment than 

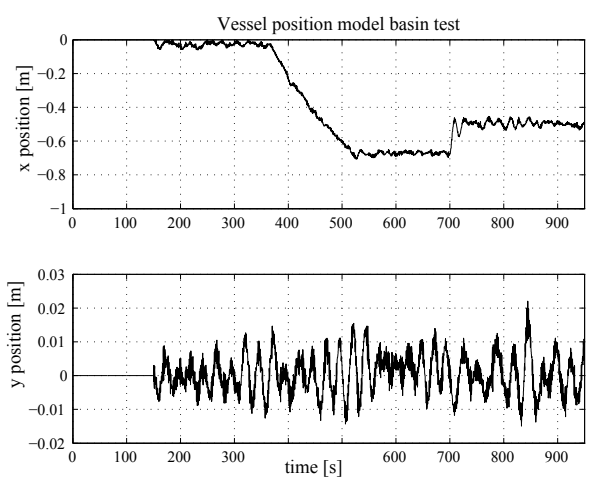

(a) Position variation from simulation
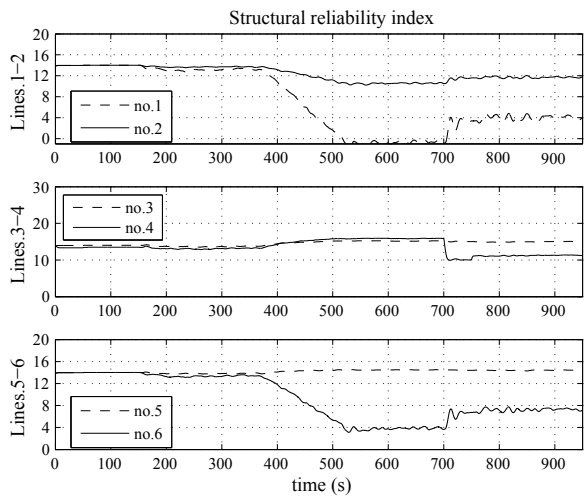

(b) Index of lines 1-6 from simulation
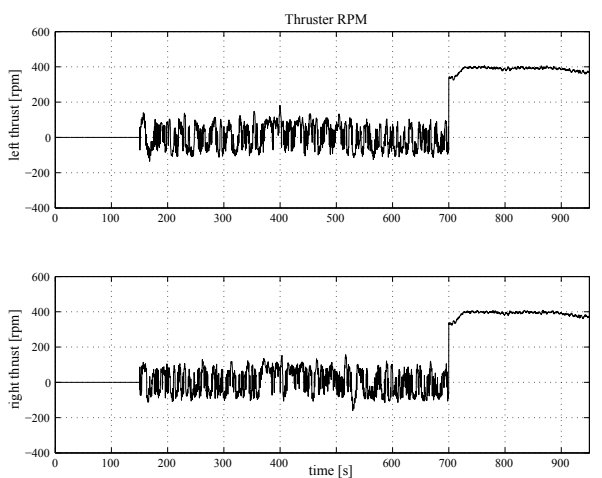

(c) Thruster RPM

Figure 13: Simulation with loss of buoyancy element in line 4 in simulations. One reason is the mounting of mooring lines in the experiment could be seen as a pendulum. There was some vibration of this pendulum in the experiment that created excess fluctuations on the body rates and hence excess thruster activity. Furthermore, there is little difference between the two sets of thruster data. The reason is that only the two aft thrusters were active during this experiment for reasons of an equipment defect. The fore thruster, that was unavailable, has a rather minor impact on vessel motions under the experiment conditions.

Line no. 1 is broken at time $t=700 \mathrm{~s}$. Figs. (14(c)) and $(14(d))$ show that the index of line no.1 is already below the critical value $\delta_{c}=4$ before $t=700 s$. It is apparent that the risk of line breakage increases and thus the line breakage is induced at the time $t=700 \mathrm{~s}$. Once the line is broken, the fault-tolerant control strategy is initiated to accommodate the effect of the fault. The most loaded line, no.6, comes to be beyond the critical index, while the other lines no.2-5 are also in a safe state and the indices of all these lines have good margins to the critical value. Both simulation and the experiment results confirm this observation.

\section{Conclusion}

This paper analysed fault diagnosis related to the mooring lines that are the key parts of a positionmooring system for a vessel. Diagnostic results were used to obtain fault-tolerant control. Fault accommodation was achieved in the control through a structural-reliability-based strategy that was shown to deal efficiently with line failure or loss of a buoyancy element. Fault diagnosis was shown to be feasible using vector-based change detection. Properties of residuals were investigated and both whitening and nonlinear compression were found necessary to meet the theoretical conditions for change detection in order to get correct probabilities of false alarms from the theoretical change detection method. Having obtained diagnostic results, a reliability index was used to calculate a shift in setpoint for the mooring system with the feature that all intact lines were kept in a safe state, even with more than one line failure, provided that thrusters could remain within their limits of control authority. The combination of diagnosis and structural reliability index based control was shown to considerably improve the safety of the mooring system. 

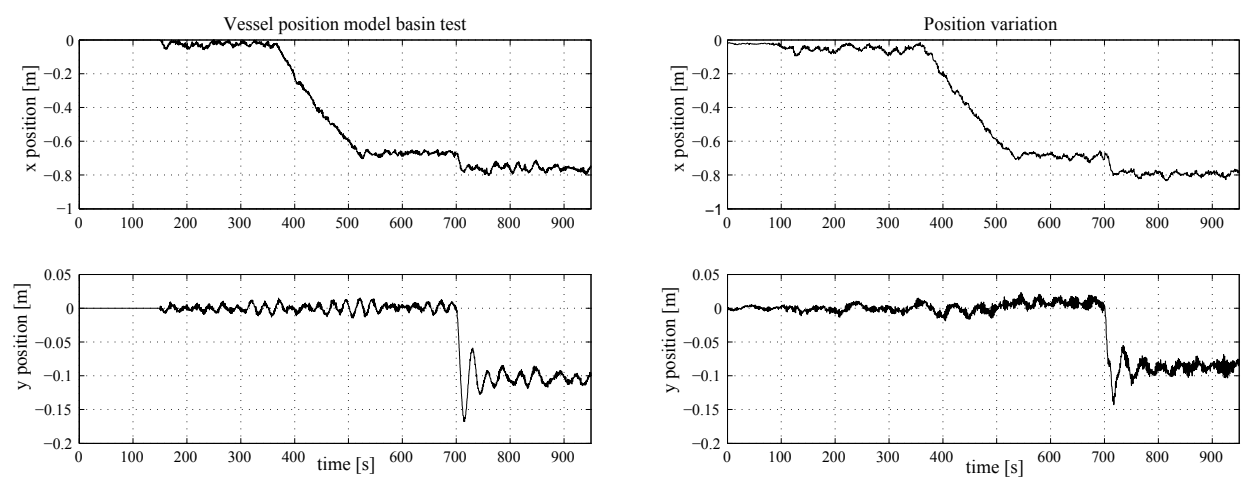

(a) Vessel motion from simulation

(b) Vessel motion from experiment
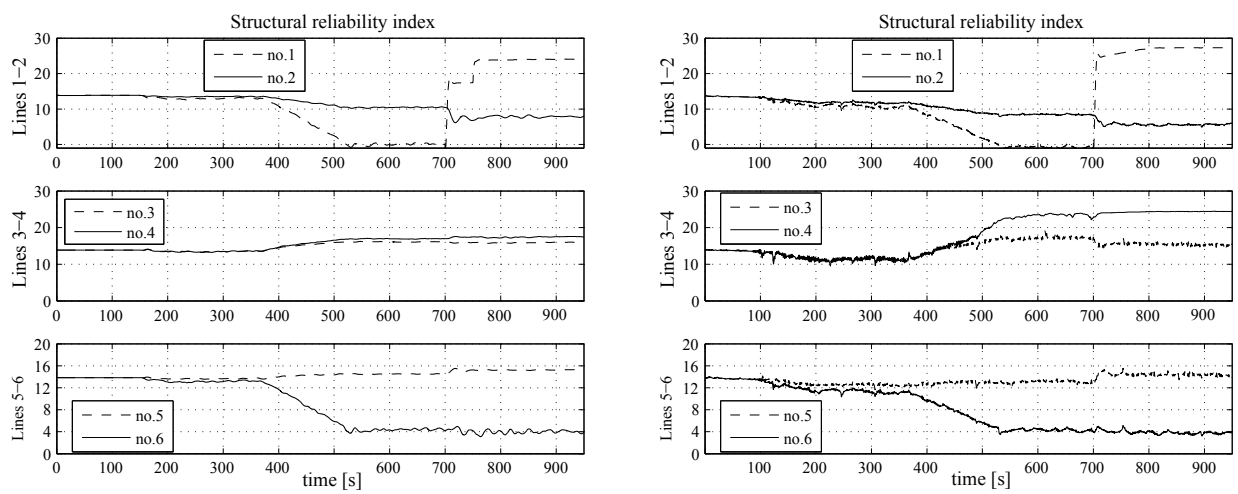

(c) Index of lines 1-6 from simulation

(d) Index of lines 1-6 from experiment
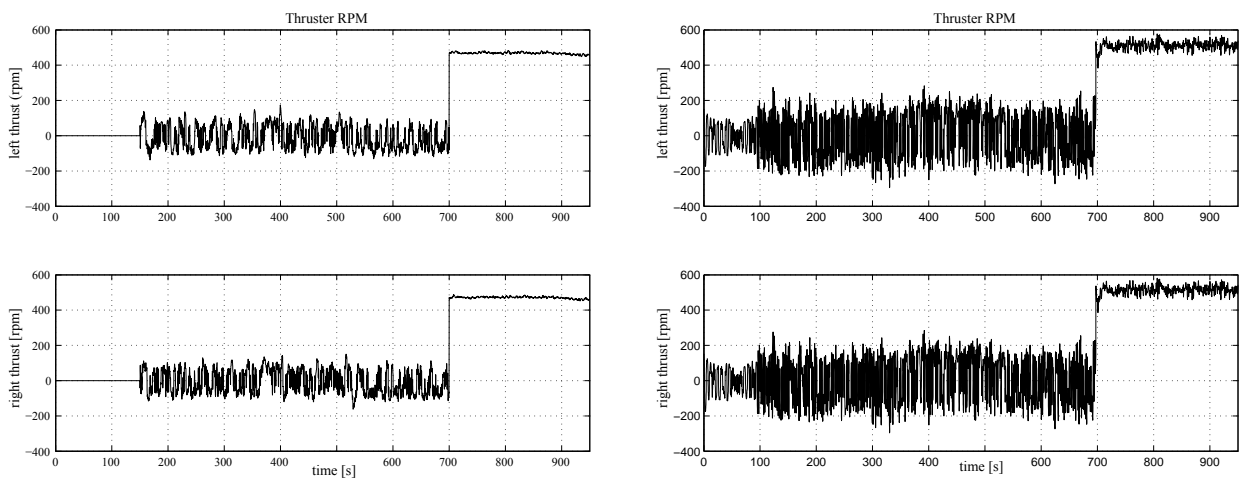

(e) Thruster RPM from simulation

(f) Thruster RPM from experiment

Figure 14: Vessel motion and structural reliability index with fault-tolerant control in simulations (left) and experiment (right) 
The approach was convincingly validated by model tank experiments.

\section{Acknowledgement}

The support of this research from the Research Council of Norway through the CeSOS and AMOS Centres of Excellence (grant 223254 for AMOS), are gratefully acknowledged. Mr. Torgeir Wahl, is gratefully acknowledged for his support during the experiments.

\section{Appendix A. Modelling of PM system}

With the structural analysis technique, the model of a system is considered as a set of constraints $\mathbf{C}=\left\{a_{1}, \ldots, a_{i}, c_{1}, \ldots c_{i}, d_{1}, \ldots, d_{i}, m_{1}, \ldots, m_{i}\right\}$ that are applied to a set of variables $\mathbf{X}=X \cup K$. $X$ denotes the set of unknown variables, $K=K_{i} \cup K_{m}$ known variables: measurements $\left(K_{m}\right)$, control input $\left(K_{i}\right)$ etc. Variables are constrained by the physical laws applied to a particular unit. With $k$ thrusters and $n$ mooring lines, the constraints are:

$$
\begin{aligned}
& a_{1}: \quad T_{1}=g_{t}\left(u_{1}, u_{2}, \ldots, u_{k}\right) \\
& a_{2}: \quad T_{2}=g_{l}\left(u_{1}, u_{2}, \ldots, u_{k}\right) \\
& a_{3}: \quad T_{3}=g_{l}\left(u_{1}, u_{2}, \ldots, u_{k}\right) \\
& c_{1}: \quad \mathbf{M} \dot{\mathbf{v}}=\mathbf{H}_{x y} \mathbf{T}\left[T_{1}, T_{2}, T_{3}\right]^{\top} \\
& +\quad\left[\begin{array}{ll}
g_{w}^{x}\left(v_{w}\right) & g_{w}^{y}\left(v_{w}\right)
\end{array}\right]^{\top} \\
& +\quad \sum_{j=1}^{n} \mathbf{A}_{m o}^{x y}(\mathbf{p}, \psi) \mathbf{T}_{m o j}^{x y}\left(\mathbf{T}_{m o j}\right) \\
& \text { - } \mathbf{D}\left[\begin{array}{ll}
\mathbf{v} & \dot{\psi}
\end{array}\right]^{\top}+\mathbf{T}_{\text {wave }}^{(1,2)} \\
& c_{2}: \quad \mathbf{I}_{z z} \ddot{\psi}=\mathbf{H}_{\psi} \mathbf{T}\left[T_{1}, T_{2}, T_{3}\right]^{\top} \\
& +\quad \sum_{j=1}^{n} \mathbf{A}_{m o}^{\psi}(\mathbf{p}, \psi) \mathbf{T}_{m o j}^{\psi}\left(\mathbf{T}_{m o j}\right) \\
& +\quad g_{w}^{\psi}\left(v_{w}\right)+\mathbf{T}_{\text {wave }}^{(3)} \\
& c_{3}: \quad \dot{\mathbf{p}}=\mathbf{A}_{v e}(\psi) \mathbf{v}+\mathbf{v}_{c} \\
& c_{4}: \quad \mathbf{p}_{G 1}=\mathbf{p}+\mathbf{R}(\phi, \theta, \psi) \mathbf{l}_{G 1} \\
& c_{5}: \quad \mathbf{p}_{G 2}=\mathbf{p}+\mathbf{R}(\phi, \theta, \psi) \mathbf{l}_{G 2} \\
& c_{6}: \quad \mathbf{p}_{H 1}=\mathbf{p}+\mathbf{R}(\phi, \theta, \psi) \mathbf{l}_{H 1} \\
& c_{2 i+5}: \quad \mathbf{T}_{m o j}=g_{m o}\left(\mathbf{p}, \psi, \mathbf{T}_{m b i}\right) \\
& c_{2 i+6}: \quad \mathbf{T}_{m b i}=g_{m b}(\mathbf{p}, \psi)
\end{aligned}
$$

$$
\begin{array}{rrl}
d_{1}: & \dot{\mathbf{v}}= & \frac{\partial}{\partial t} \mathbf{v} \\
d_{2}: & \dot{\mathbf{p}}= & \frac{\partial}{\partial t} \mathbf{p} \\
d_{3}: & \dot{\psi}= & \frac{\partial}{\partial t} \psi \\
d_{4}: & \ddot{\psi}= & \frac{\partial}{\partial t} \dot{\psi} \\
m_{1 . .} m_{3}: & \psi_{1 . \cdot 3}= & \psi \\
m_{4}: & \mathbf{p}_{G 1}^{m}= & \mathbf{p}_{G 1} \\
m_{5}: & \mathbf{p}_{G 2}^{m}= & \mathbf{p}_{G 2} \\
m_{6}: & \mathbf{p}_{H 1}^{m}= & \mathbf{p}_{H 1} \\
m_{7 . . m_{9}:}: & \mathbf{q}_{1 \cdot .3}= & {\left[\begin{array}{ll}
z & \phi
\end{array}\right]} \\
m_{10}: & \mathbf{v}_{m}= & \mathbf{v} \\
m_{11,12}: & \mathbf{w}_{m 1, m 2}= & \mathbf{v}_{w} \\
m_{13}: & \mathbf{c}_{m}= & \mathbf{v}_{c} \\
m_{13+j}: & \mathbf{T}_{m j}= & \mathbf{T}_{m o j},
\end{array}
$$

where $a_{i}$ are the constraint of the thruster input, $c_{i}$ algebraic constraints, $d_{i}$ differential constraints, $m_{i}$ measurements, and $\mathbf{M}$ is the mass matrix including added mass, $\mathbf{D}$ is the damping matrix, $\mathbf{I}_{z z}$ is the inertia moment for yaw, $\mathbf{T}$ is the thruster configuration matrix, $\mathbf{H}_{x y}$ is the projection matrix for surge and sway, $\mathbf{H}_{\psi}$ is that for yaw, $\mathbf{A}_{m o}^{x y}, \mathbf{A}_{m o}^{\psi}$ is a transformation matrix for horizontal mooring line tension from Earth-fixed to body-fixed frame, $\mathbf{A}_{v e}(\psi)$ is a transformation matrix for vessel velocity from Earth-fixed to body-fixed frame, $\mathbf{R}(\phi, \theta, \psi)$ is the transformation from position reference system to vessel coordinate origin, and $g_{w}^{x}\left(v_{w}\right)$, $g_{w}^{y}\left(v_{w}\right), g_{w}^{\psi}\left(v_{w}\right)$ are the wind forces in surge, sway and the moment in yaw. Wave induced forces on the hull are represented by $\mathbf{T}_{\text {wave }}^{(1,2)}$, and their moment by $\mathbf{T}_{\text {wave }}^{(3)}$. Wave and current forces on the mooring line themselves have been disregarded, in accordance with assumptions made in [40].

Categorising variables in the constraints as belonging to the sets $X$ unknown, $K_{i}$ input and measurements $K_{m}$, the variables are be separated as:

$$
\begin{aligned}
X= & \left\{T_{1}, T_{2}, T_{3}, \mathbf{T}_{m b i}, \mathbf{T}_{m o j}, \mathbf{T}_{\text {wave }}, \mathbf{p}_{G 1},\right. \\
& \mathbf{p}_{G 2}, \mathbf{p}_{H 1}, \mathbf{v}, \dot{\mathbf{v}}, \psi, \dot{\psi}, \ddot{\psi},, \mathbf{p}, \dot{\mathbf{p}}, \theta, \phi, \\
& \left.\mathbf{v}_{c}, \mathbf{v}_{w}\right\} \\
K_{i}= & \left\{u_{1}, u_{2}, \ldots, u_{k}\right\} \\
K_{m}= & \left\{\psi_{1}, \psi_{2}, \psi_{3}, \mathbf{p}_{G 1}^{m}, \mathbf{p}_{G 2}^{m}, \mathbf{p}_{H 1}^{m}, \mathbf{q}_{1},\right. \\
& \left.\mathbf{q}_{2}, \mathbf{q}_{3}, \mathbf{v}_{m},, \mathbf{w}_{m 1}, \mathbf{w}_{m 2}, \mathbf{c}_{m}, \mathbf{T}_{m j}\right\} .
\end{aligned}
$$

The modelling here presents the normal behaviour, and diagnostic algorithms will be designed 
to detect deviation from normal, where the occurrence of full or partial failure of mooring lines can be detected and counteracted by thruster-assisted position control.

\section{Appendix B. Design of control plant model}

The process plant model is detailed and accurate, which is used here to do the structural analysis for the PM system. The detailed design of the process plant can refer to [26], [41] and [11]. The control plant model is a simplified model used for design and analysis of the observer and controller.

The slowly varying environmental forces due to wind, current and waves are modelled as a bias term b in a first-order Markov process:

$$
\dot{\mathbf{b}}=-\mathbf{T}^{-1} \mathbf{b}+\mathbf{\Psi} \mathbf{n}
$$

where $\mathbf{b} \in \mathbb{R}^{3}$ is the vector of bias force and moment, $\mathbf{n} \in \mathbb{R}^{3}$ is the vector of white noise, $\mathbf{T} \in \mathbb{R}^{3 \times 3}$ is the diagonal matrix of positive bias time constant, and $\boldsymbol{\Psi} \in \mathbb{R}^{3 \times 3}$ is the diagonal matrix scaling the amplitude of $n$. This model is usually used to describe slowly varying environmental force and moment due to second-order wave drift, current, wind and un-modelled dynamics.

The WF model used is a second-order linear model driven by white noise:

$$
\begin{aligned}
\dot{\boldsymbol{\xi}}_{\omega} & =\mathbf{A}_{\omega} \boldsymbol{\xi}_{\omega}+\boldsymbol{\Sigma}_{\omega} \boldsymbol{\omega}_{\omega} \\
\boldsymbol{\eta}_{w} & =\Gamma_{\omega} \boldsymbol{\xi}_{\omega}
\end{aligned}
$$

where $\boldsymbol{\xi}_{\omega} \in \mathbb{R}^{6}$ is the state of the WF model, and $\mathbf{A}_{\omega} \in \mathbb{R}^{6 \times 6}$ is assumed to be stable and describe the first-order WF-induced motion as:

$$
\mathbf{A}_{\omega}=\left[\begin{array}{cc}
\mathbf{0}^{3 \times 3} & \mathbf{I}^{3 \times 3} \\
-\boldsymbol{\Omega}^{2} & -2 \boldsymbol{\Lambda} \boldsymbol{\Omega}
\end{array}\right]
$$

and $\boldsymbol{\Omega}=\operatorname{diag}\left(\omega_{01}, \omega_{02}, \omega_{03}\right)$ is the diagonal matrix containing the dominating frequencies of the response of the vessel subjected to the first-order wave loads; and $\boldsymbol{\Lambda}=\operatorname{diag}\left(\lambda_{1}, \lambda_{2}, \lambda_{3}\right)$ is the diagonal matrix containing the damping ratios.

Assuming anchor lines are fixed un-stretched lengths, the generalised mooring-line forces at a working point are approximated by a linearised static restoring and damping:

$$
\boldsymbol{\tau}_{m o}=-\mathbf{R}^{\boldsymbol{\top}}(\psi) \mathbf{G}_{m o}\left(\boldsymbol{\eta}-\boldsymbol{\eta}_{0}\right)-\mathbf{D}_{m o} \boldsymbol{v}
$$

The stiffness matrix $\mathbf{G}_{m o}$ is approximated by the first-order expansion of the static force at the position $\boldsymbol{\eta}_{0}=0$, and the damping matrix $\mathbf{D}_{m o}$ is calculated by a least squares fit [42].
Non-linear damping components $\mathbf{D}_{N L}\left(\boldsymbol{\nu}_{r}, \gamma_{r}\right) \boldsymbol{\nu}_{r}$ and $\mathbf{C}_{R B}(\boldsymbol{\nu}) \boldsymbol{\nu}, \mathbf{C}_{A}\left(\boldsymbol{\nu}_{r}\right) \boldsymbol{\nu}_{r}$ in the low-frequency dynamics are usually small since the floater velocity is quite small for the station keeping motion. Then the LF model together with the kinematics can be simplified to:

$$
\begin{aligned}
\dot{\boldsymbol{\eta}} & =\mathbf{R}(\psi) \boldsymbol{\nu} \\
\mathbf{M} \dot{\boldsymbol{\nu}} & =\mathbf{R}^{\boldsymbol{\top}}(\psi) \mathbf{b}-\mathbf{R}^{\boldsymbol{\top}}(\psi) \mathbf{G}_{m o} \boldsymbol{\eta}+\boldsymbol{\tau}_{t h r}-\mathbf{D} \boldsymbol{\nu} \\
\dot{\mathbf{b}} & =-\mathbf{T}^{-1} \mathbf{b}+\mathbf{\Psi} \mathbf{n}
\end{aligned}
$$

where $\boldsymbol{\eta}=\left[\begin{array}{lll}x & y & z\end{array}\right]^{\top} \in \mathbb{R}^{3}$ is the LF position in the Earth-fixed frame $(x, y, z$ are the north, south and yaw, respectively) and $\mathbf{v}=\left[\begin{array}{lll}u & v & r\end{array}\right]^{\top} \in \mathbb{R}^{3}$ is the LF velocity in the body-fixed frame $(u, v, r$ are velocities in surge, sway and yaw). The damping matrix $\mathbf{D}=\mathbf{D}_{L}+\mathbf{D}_{m o}$ includes the mooring system damping $\mathbf{D}_{m o}$.

The output of the control plant model $\mathbf{y} \in \mathbb{R}^{3}$ is position and heading of the vessel, a superposition of the WF and LF motions:

$$
\mathbf{y}=\boldsymbol{\eta}+\Gamma_{\omega} \boldsymbol{\xi}_{\omega}+\mathbf{v}
$$

where $\mathbf{v} \in \mathbb{R}^{3}$ is zero-mean Gaussian noise.

\section{Appendix B.1. Observer design}

Following [3], a non-linear passive observer is found using the control plant model,

$$
\begin{aligned}
\dot{\hat{\boldsymbol{\xi}}}_{\omega}= & \mathbf{A}_{\omega} \hat{\boldsymbol{\xi}}_{\omega}+\mathbf{K}_{1} \tilde{\mathbf{y}} \\
\dot{\hat{\boldsymbol{\eta}}}= & \mathbf{R}(\psi) \hat{\boldsymbol{\nu}}+\mathbf{K}_{2} \tilde{\mathbf{y}} \\
\mathbf{M} \dot{\hat{\boldsymbol{\nu}}=} & \mathbf{R}^{\boldsymbol{\top}}(\psi) \hat{\mathbf{b}}-\mathbf{D} \hat{\boldsymbol{\nu}}-\mathbf{R}^{\boldsymbol{\top}}(\psi) \mathbf{G}_{m o} \hat{\boldsymbol{\eta}} \\
& +\boldsymbol{\tau}_{t h r}+\mathbf{K}_{3} \mathbf{R}^{\top}(\psi) \tilde{\mathbf{y}} \\
\dot{\mathbf{b}}= & -\mathbf{T}^{-1} \mathbf{b}++\mathbf{K}_{4} \tilde{\mathbf{y}} \\
\hat{\mathbf{y}}= & \hat{\boldsymbol{\eta}}+\Gamma_{\omega} \hat{\boldsymbol{\xi}}_{\omega}
\end{aligned}
$$

where $\tilde{\mathbf{y}}=\mathbf{y}-\hat{\mathbf{y}}$ is the estimation error; and $\mathbf{K}_{1}, \mathbf{K}_{2}, \mathbf{K}_{3}, \mathbf{K}_{4}$ are observer gain matrices, see [3]. The stability analysis and tuning for the observer are very similar to those addressed in [3] except for two new terms: the damping term $\mathbf{D}_{m o}$ and the stiffness term $\mathbf{G}_{m o}$. For increasing damping and stiffness, the system would become more stable and the specific passive observer stability analysis is described in [3].

\section{Appendix B.2. Controller design}

The controller's objective is to maintain the vessel's position and keep the mooring system in a safe state. The control action counteracts the lowfrequency part of vessel motion from current, wind 
and second order mean and slowly varying wave loads. Control at the wave frequency motion is avoided. A multi-variable PID controller is employed:

$$
\begin{aligned}
\boldsymbol{\tau}_{t h r}= & -\mathbf{K}_{i} \mathbf{R}^{T}(\varphi) \int_{0}^{t} \hat{\boldsymbol{\eta}}(\boldsymbol{\tau})_{e} d \tau \\
& -\mathbf{K}_{p} \mathbf{R}^{T}(\varphi) \hat{\boldsymbol{\eta}}_{e}-\mathbf{K}_{d} \hat{\boldsymbol{\nu}}_{e}
\end{aligned}
$$

where $\hat{\boldsymbol{\eta}}_{e}=\boldsymbol{\eta}-\boldsymbol{\eta}_{d} ; \hat{\boldsymbol{\nu}}_{e}=\boldsymbol{\nu}-\boldsymbol{\nu}_{d} ; \boldsymbol{\eta}_{d}$ and $\boldsymbol{\nu}_{d}$ are the desired position and velocity vectors respectively; and $\mathbf{K}_{d}, \mathbf{K}_{i}$ and $\mathbf{K}_{d} \in \mathbb{R}^{3 \times 3}$ are non-negative control gain matrices. $\psi$ is the measured heading. The wind feed-forward controller, acceleration feedback, and roll-pitch damping could be used in addition. The functionality of different control modes refers to [7] and combination with reliability index monitoring was considered in [43].

\section{Appendix C. Set-point chasing algorithm}

The algorithm for set-point chasing was derived in [18], the main result is repeated here for completeness and convenience. The set-point is calculated based on the quadratic cost function in Eq. (28). The optimal increment of the vessel position and its direction are,

$$
\begin{aligned}
\Delta r & =\frac{K_{11} \sin \beta+K_{12} \cos \beta}{K_{21} \sin ^{2} \beta+K_{b}+K_{23} \cos ^{2} \beta} \\
K_{b} & =2 K_{22} \sin \beta \cos \beta \\
\beta & =\operatorname{tg}^{-1} \frac{K_{11} K_{23}-K_{12} K_{22}}{K_{21} K_{12}-K_{11} K_{22}}
\end{aligned}
$$

where the time-varying parameters $K_{11}$ to $K_{23}$ depend on the mooring-line angles $\beta_{i 0}$, physical parameters and the reliability indexes as follows:

$$
\begin{aligned}
K_{11}= & \kappa_{1}\left(\delta_{c 1}-\delta_{1}\right) \cos \beta_{1 o}+\kappa_{2}\left(\delta_{c 2}-\delta_{2}\right) \cos \\
& \beta_{2 o}+\cdots+\kappa_{n}\left(\delta_{c n}-\delta_{n}\right) \cos \beta_{n o} \\
K_{12}= & \kappa_{1}\left(\delta_{c 1}-\delta_{1}\right) \sin \beta_{1 o}+\kappa_{2}\left(\delta_{c 2}-\delta_{2}\right) \sin \\
& \beta_{2 o}+\cdots+\kappa_{n}\left(\delta_{c n}-\delta_{n}\right) \sin \beta_{n o} \\
K_{21}= & \vartheta_{1} \cos ^{2} \beta_{1 o}+\vartheta_{2} \cos ^{2} \beta_{2 o} \\
& +\cdots+\vartheta_{n} \cos ^{2} \beta_{n o} \\
K_{22}= & \vartheta_{1} \sin \beta_{1 o} \cos \beta_{1 o}+\vartheta_{2} \sin \beta_{2 o} \cos \beta_{2 o} \\
& +\cdots+\vartheta_{n} \sin \beta_{n o} \cos \beta_{n o} \\
K_{23}= & \vartheta_{1} \sin ^{2} \beta_{1 o}+\vartheta_{2} \sin ^{2} \beta_{2 o} \\
& +\cdots+\vartheta_{n} \sin ^{2} \beta_{n o}
\end{aligned}
$$

where $\kappa_{i}$ and $\vartheta_{i}$ are material parameters for line $i$. For detailed deduction and description about the parameters refer to [11].

\section{References}

[1] Balchen, J., Jenssen, N., Mathisen, E., and Sælid, S., 1980. "A dynamic positioning system based on kalman filtering and optimal control". Modelling, Identification and Control, pp. 135-163.

[2] Sørensen, A., Sagatun, S., and Fossen, T., 1996. "Design of a dynamic position system using modelbased control". Control Engineering Practice, pp. 359368.

[3] Fossen, T., and Strand., J., 1999. "Passive nonlinear observer design for ships using lyapunov methods: Full scale experiments with a supply vessel". Automatica, pp. $3-16$.

[4] Strand, J., Sørensen, A., and Fossen, T., 1998. "Design of automatic thruster assisted position mooring systems for ships". Modelling, Identification and Control, pp. $61-75$.

[5] Aamo, O., and Fossen, T., 1999. "Controlling line tension in thruster assisted mooring systems". Proceedings of IEEE International Conference on Control Applications in Marine Systems (CAMS), Nertherland, pp. 1104-1109.

[6] Aamo, O., and Fossen, T., 2001. "Finite element modelling of moored vessels". Mathematical and Computer Modelling of Dynamical Systems, pp. 47-75.

[7] Nguyen, D., and Sørensen, A., 2009. "Switched control for thruster-assisted position mooring". Control Engineering Practice, pp. 985-994.

[8] Wang, Y., Zou, C., Ding, F., Dou, X., Ma, Y., and Liu, Y., 2014. "Structural reliability based dynamic positioning of turret-moored fpsos in extreme seas". Mathematical Problems in Engineering.

[9] DNV, October, 2008. "Position mooring". Offshore Standard, DNV-OS-E301.

[10] Gao, Z., and Moan, T., 2007. "Sensitivity study of extreme value and fatigue damage of line tension in mooring system with one line failure under varying annual environmental condition". Proceedings of The 17th International Offshore and Polar Engineering Conference (ISOPE).

[11] Fang, S., Leira, B. J., and Blanke, M., 2013. "Position mooring control based on a structural reliability criterion". Structural Safety, 41, pp. 97-106.

[12] Baker, J., and McCafferty, D., 2005. "Accident database review of human element concerns: what do the results mean for classification". In Proceedings of the international conference on human factors in ship design and operation, London, RINA.

[13] Blanke, M., Kinnaert, M., Lunze, J., and Staroswiecki, M., 2006. Diagnosis and Fault-Tolerant Control. Springer, Heidelberg.

[14] Blanke, M., 2005. "Diagnosis and fault-tolerant control for ship station keeping". Proc. 13th Mediterranean Conference on Control and Automation.

[15] Blanke, M., 2006. "Fault-tolerant sensor fusion for marine navigation". In Proceedings of the 7 th IFAC conference on maneuvering and control of marine craft (MCMC), Lisbon, Portugal.

[16] Nguyen, D., Blanke, M., and Sørensen, A., 2007. "Daignosis and fault-tolerant control for thrusterassisted position mooring". In Proc. IFAC Conference on Control Applications in Marine Systems.

[17] Nguyen, T. D., and Blanke, M., 2015. "Fault-tolerant positioning control for offshore vessels with thruster and 
mooring actuation". Journal of Adaptive Control and Signal Processing, p. Submitted.

[18] Fang, S., and Blanke, M., 2011. "Fault monitoring and fault recovery control for position moored tanker". International Journal of Applied Mathematics and Computer Science, 21 (3).

[19] Blanke, M., Fang, S., Galeazzi, R., and Leira, B. J. 2012. "Statistical change detection for diagnosis of buoyancy element defects on moored floating vessels". In Proc. IFAC Safeprocess, Mexico, IFAC-Papers Online.

[20] Leira, B. J., Chen, Q., Sørensen, A., and Larsen, C. M. 2001. "Modelling of riser response for dp control". In Proceedings of the 20th international conference on Ocean, Offshore and Artic Engineering (OMAE), pp. 383-391.

[21] Leira, B. J., Sørensen, A., and Larsen, C., 2004. "A reliability-based control algorithm for dynamic positioning for floating vessels". Structural Safety, 26, pp. 1-28.

[22] Berntsen, P., Aamo, O., and Leira, B., 2008. "Structural reliability-based control of moored interconnected structures". Control Engineering Practice, pp. 495-504.

[23] Berntsen, P., Aamo, O., and Leira, B., 2008. "Thruster assisted position mooring based on structural reliability". Int. Journal of Control, 81, pp. 1408-1416.

[24] IMO, 1994. Guidelines for vessels with dynamic positioning systems, The International Marine Contractors Association, IMO MSC Circular 645. IMO.

[25] DNV, 2011. "Dynamic positioning systems". Rules for Classification of Ships, p. Part 6 Chapter 7.

[26] Fossen, T. I., 2002. Marine Control System. Marine Cybernetics, Norway.

[27] Blanke, M., and Staroswiecki, M., 2006. "Structural design of systems with safe behaviour under single and multiple faults". In Proc. IFAC Safeprocess Symposium 2006.

[28] Huber, P. J., 1981. Robust Statistics. J. Wiley \& Sons.

[29] Basseville, M., and Nikiforov, I. V., 1993. Detection of Abrupt Changes: Theory and Application. Information and System Science. Prentice Hall, New York.

[30] Kay, S., 1998. Fundamentals of Statistical Signal Processing, Volume 2: Detection Theory. Prentice Hall.

[31] Travé-Massuyès, L., 2014. "Bridging control and artificial intelligence theories for diagnosis: A survey". Engineering Applications of Artificial Intelligence, 27, pp. 1-16.

[32] Svärd, C., Nyberg, M., Frisk, E., and Krysander, M. 2013. "Automotive engine FDI by application of an automated model-based and data-driven design methodology". Control Engineering Practice, 4, pp. 455472.

[33] Svärd, C., Nyberg, M., Frisk, E., and Krysander, M., 2014. "Data-driven and adaptive statistical residual evaluation for fault detection with an automotive application". Mechanical Systems and Signal Processing, 45 (1), pp. 170-192.

[34] Nguyen, D., and Sørensen, A., 2007. "Setpoint chasing for thruster-assisted position mooring". In Proc. 26th Int. Conf. on Ocean, Offshore and Artic Engineering.

[35] Triantafyllou, M., 1990. "Cable mechanics with marine applications". Lecture notes, Department of Ocean Engineering, Massachusetts Institute of Technology, Cambridge, $M A$.

[36] Triantafyllou, M., 1994. "Cable mechanics for moored floating systems". Proceddings of BOSS, pp. 67-77.

[37] Madsen, H., Krenk, S., and Lind, N., 1986. Methods of Structrual Safety. Prentice-Hall, New York, USA.

[38] MSS, 2010. "Marine system simulator". http://www.marinecontrol.org.

[39] Guedes Soares, C., Fonseca, N., and Pascoal, R., 2005. "Experimental and numerical study of the motion of a turret-moored fpso in waves". Journal of Offshore Mechanics and Arctic Engineering, 10, pp. 197-204.

[40] Berntsen, P. I. B., Aamo, O. M., and Leira, B. J., 2009. "Ensuring mooring line integrity by dynamic positioning: Controller design and experimental tests". Automatica, 45, pp. 1285-1190.

[41] Nguyen, D., and Sørensen, A., 2009. "Setpoint chasing for thruster-assisted position mooring". IEEE Journal of Oceanic Engieering, 34(4), pp. 548-558.

[42] Ormberg, H., and Larsen, K., 1998. "Coupled analysis of floater motion and mooring dynamics for a turretmoored ship". Applied Ocean Research, 20, pp. 55-67.

[43] Berntsen, P., Aamo, O., and Leira, B., 2006. "Dynamic positioning of moored vessels based on structure reliability". In Proc. 45th IEEE Conf. on Decision and Control (CDC), San Diego, pp. 5906-5911. 